\title{
Tunneling Between Parallel Two-Dimensional Electron Gases
}

\author{
N. Turner, J. T. Nicholls, E. H. Linfield, K. M. Brown, G. A. C. Jones, D. A. Ritchie \\ Cavendish Laboratory, Madingley Road, Cambridge CB3 OHE, UK
}

(February 25, 2018)

\begin{abstract}
The tunneling between two parallel two-dimensional electron gases has been investigated as a function of temperature $T$, carrier density $n$, and the applied perpendicular magnetic field $B$. In zero magnetic field the equilibrium resonant lineshape is Lorentzian, reflecting the Lorentzian form of the spectral functions within each layer. From the width of the tunneling resonance the lifetime of the electrons within a 2DEG has been measured as a function of $n$ and $T$, giving information about the density dependence of the electronimpurity scattering and the temperature dependence of the electron-electron scattering. In a magnetic field there is a general suppression of equilibrium tunneling for fields above $B=0.6 \mathrm{~T}$. A gap in the tunneling density of states has been measured over a wide range of magnetic fields and filling factors, and various theoretical predictions have been examined. In a strong magnetic field, when there is only one partially filled Landau level in each layer, the temperature dependence of the conductance characteristics has been modeled with a double-Gaussian spectral density.
\end{abstract}




\section{INTRODUCTION}

In addition to conserving energy $E$, resonant tunneling between two parallel twodimensional electron gases (2DEGs) requires the conservation of the in-plane momentum $\hbar \boldsymbol{k}$. In zero magnetic field, the conservation of both quantities allows the investigation of the broadening of the electronic states within an individual 2DEG, in a way that is not possible with conventional transport measurements. For example, recent experimental studiest have shown that the lifetime of the two-dimensional electrons can be measured directly by tunneling spectroscopy. The lifetime broadening is characterized by the electron spectral function $A(\boldsymbol{k}, E)$, which describes the probability that an electron in a particular $\boldsymbol{k}$-state has energy $E$. In general, $A(\boldsymbol{k}, E)$ is strongly peaked near the free-particle energy $E_{\boldsymbol{k}}=\hbar^{2} k^{2} / 2 m^{\star}$, with a width $\Gamma$ determined by the lifetime $\tau$ of the electron.

In a strong perpendicular magnetic field, when the Landau level filling factor $\nu=h n / e B$ is less than unity, it is assumed that the electron traverses the tunnel barrier between the two 2DEGs in a much shorter timescale than the charge rearrangement around the injected electron (and around the hole left behind in the emitter layer). The energies of the injection and extraction processes create a gap in the tunneling density of states (DOS). Many-body theories predict 3 that for a fixed filling factor, $\nu<1$, the width of the energy gap is

approximately $0.4 e^{2} / 4 \pi \epsilon l_{B}$ (where $l_{B}=\sqrt{\hbar / e B}$ is the magnetic length), or if the electron liquid forms a regular lattice 6 the gap energy is $e^{2} / 4 \pi \epsilon a$ (where $a=1 / \sqrt{n \pi}$ is the interelectron spacing within a layer). At fixed filling factor both predicted forms for the gap have the same $\sqrt{B}$ dependence with magnetic field. In an earlier paper we reported tunneling characteristics in the high magnetic field regime $(\nu<1)$. The theoretical predictions do not match the measured linear field dependence, $\Delta \approx 0.44 \hbar \omega_{\mathrm{c}}$ (where $\hbar \omega_{\mathrm{c}}=\hbar e B / \mathrm{m}^{\star}$ is the cyclotron energy), that we have observed 0,8 in high magnetic fields both at fixed filling factor and constant carrier density.

From tunneling measurements of 2D-2D samples with different barrier widths, Eisenstein et al. 9 argue that the high field gap energy is largely determined by the in-plane Coulomb energy, modified by an interlayer excitonic binding energy. The form of the gap used to fit their data was $\Delta_{\mathrm{E}}=f(\nu) e^{2} / 4 \pi \epsilon a-0.4 e^{2} / 4 \pi \epsilon d$, where $f(\nu)$ is some universal function of filling factor, and $d$ is the quantum well center-to-center separation. Although the interpretation of our data is different to that of Eisenstein et al 9 (there is no universal $f(\nu)$ which allows us to plot our high field data in this form), we measure gaps of comparable energy in lower mobility, but otherwise similar GaAs/AlGaAs double-layer structures.

In a 2D-3D tunneling system Ashoori et al.10 measured a filling factor independent suppression of tunneling for $\nu>1$. The temperature dependence of the conductance was fitted to a model with a linear variation of the DOS about the Fermi energy $E_{\mathrm{F}}$. The half-width at half-depth of the gap was measured to be linear in magnetic field, with a dependence $\Delta_{\mathrm{A}} \approx \hbar \omega_{\mathrm{c}} / 20$. Taking into account the difference in the two tunneling systems and the definitions of $\Delta_{\mathrm{A}}$ and $\Delta$, the two gap widths can be argued 11 to be comparable.

Here we consolidate earlier work 812 at zero and high magnetic fields, and present data taken at intermediate fields. The rest of this paper is organized as follows. The samples and measurement details are described in Sec. [1]. The basic tunneling formalism is reviewed in Sec. III, showing how the tunneling conductance is determined by the underlying electron spectral functions within each 2DEG. In Sec. IV we present tunneling measurements in zero 
magnetic field, investigating the various scattering mechanisms which are responsible for the linewidth of the resonance. The tunneling properties in a perpendicular magnetic field are investigated in Secs. $\nabla$ and $\nabla 1$.

\section{EXPERIMENTAL DETAILS}

The measurements presented in this paper were obtained from two samples, C751 and C770, each consisting of two modulation-doped $180 \AA$ wide GaAs quantum wells separated by a $100 \AA(\mathrm{C} 770)$ or $125 \AA(\mathrm{C} 751) \mathrm{Al}_{0.33} \mathrm{Ga}_{0.67} \mathrm{As}$ barrier. The double-well structures were grown by molecular beam epitaxy on an $\mathrm{n}^{+}$epilayer, which had previously been patterned by a focussed Ga ion beam to form backgates.3 Optical lithography was used to fabricate a mesa, Ohmic contacts, and to define surface Schottky gates. Small front (back) gates near the Ohmic contacts were used to selectively deplete the upper (lower) 2DEG, forming an independent contact to the lower (upper) layer. The two 2DEGs overlap in a $100 \mu \mathrm{m} \times 150 \mu \mathrm{m}$ tunneling region. The as-grown carrier densities of the upper and lower layers in the tunneling region are $n_{1} \approx 3 \times 10^{11} \mathrm{~cm}^{-2}$ and $n_{2} \approx 2 \times 10^{11} \mathrm{~cm}^{-2}$. The corresponding, as-grown, low-temperature, mobilities of the two layers are $\mu_{1} \approx 8 \times 10^{5} \mathrm{~cm}^{2} / \mathrm{Vs}$ and $\mu_{2} \approx 2 \times 10^{5} \mathrm{~cm}^{2} / \mathrm{Vs}$. The carrier densities in the tunneling region are independently controlled by voltages $V_{\mathrm{g}_{1}}$ and $V_{\mathrm{g}_{2}}$ applied to large area front- and backgates.

Two-terminal conductance measurements of the sequential depopulation of Landau levels (LLs) in each layer were used to determine the relationship between $V_{\mathrm{g}_{1,2}}$ and the corresponding carrier density $n_{1,2}$. For example, in $\mathrm{C} 770$ the top layer has a density $n_{1}=\left(7.1 V_{\mathrm{g}_{1}} / \mathrm{V}+2.9\right) \times 10^{11} \mathrm{~cm}^{-2}$, where $-0.4 \leq V_{\mathrm{g}_{1}} \leq 0.35 \mathrm{~V}$. The carrier density at $V_{\mathrm{g}_{1}}=0$ can vary by as much as $10 \%$ from one sample cooldown to another. By contrast, the constant of proportionality (7.1) between $V_{\mathrm{g}_{1}}$ and $n_{1}$ is always the same, and is in good agreement with a model where the top 2DEG and the front gate form a parallel plate capacitor of separation $1000 \AA$.

The differential tunneling conductance $G=d I / d V_{\text {sd }}$ was measured using constant voltage $(0.1 \mathrm{mV})$ ac lockin techniques as a function of carrier density $n$, temperature $T$, and applied dc interlayer source-drain voltage $V_{\text {sd }}$. For measurements in a perpendicular magnetic field $B$ the carrier densities were always matched, and the given values of $\nu$ refer to the filling factors in each layer.

\section{TUNNELING FORMALISM}

Tunneling accesses the single-particle DOS (the DOS available for the addition of another electron), compared to conventional transport measurements which provide information about the DOS available for the excitation of an electron from the Fermi sea into a conducting state. Zheng and MacDonald 14 showed theoretically that 2D-2D tunneling characteristics in zero magnetic field can be used to determine the spectral function of an electron. Within layer $i[i=1,2$ (upper, lower)] scattering introduces an energy broadening $\Gamma_{i}=\hbar / 2 \tau_{i}$, where $\tau_{i}$ is the scattering time. In the Born approximation the spectral function

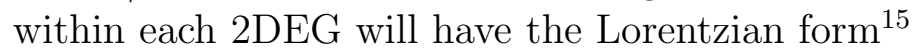




$$
A_{i}(\boldsymbol{k}, E) \propto \frac{1}{\Gamma_{i}^{2}+\left(\frac{\hbar^{2} k^{2}}{2 m^{\star}}-E\right)^{2}},
$$

where $E$ is measured from the bottom of the $2 \mathrm{D}$ subband. Using standard notation 6 the tunneling current $I\left(V_{\mathrm{sd}}\right)$ between the two layers is calculated from the two spectral functions to be

$$
\begin{aligned}
I\left(V_{\mathrm{sd}}\right) \propto & \int d^{2} \boldsymbol{k}_{1} \int d^{2} \boldsymbol{k}_{2}\left|T_{\boldsymbol{k}_{1}, \boldsymbol{k}_{2}}\right|^{2} \int d E \\
& A_{1}\left(\boldsymbol{k}_{1}, E-E_{0_{1}}\right) A_{2}\left(\boldsymbol{k}_{2}, E-E_{0_{2}}\right) \\
& \times\left[f\left(E-\mu_{1}, T\right)-f\left(E-\mu_{2}, T\right)\right],
\end{aligned}
$$

where $f(E, T)=1 /\left(1+\exp \left(E / k_{\mathrm{B}} T\right)\right)$ is the Fermi distribution function. The chemical potentials $\mu_{i}$ and the $2 \mathrm{D}$ subband energies $E_{0_{i}}$ are defined in Fig. 1 .

The integral is simplified by the following assumptions: the absolute conservation of momentum (such that the interlayer tunneling matrix element $T_{\boldsymbol{k}_{1}, \boldsymbol{k}_{2}}$ is proportional to $\left.\delta_{\boldsymbol{k}_{1}, \boldsymbol{k}_{2}}\right)$, low temperature $\left(k_{\mathrm{B}} T \ll \Gamma\right.$, with Fermi functions approximated by step functions), and weak disorder $\left(\Gamma \ll E_{\mathrm{F}}\right)$. Using these assumptions, substitution of Lorentzian spectral functions into Eq. 2 gives the tunneling current

$$
I\left(V_{\mathrm{sd}}\right)=\frac{G_{0} \Gamma^{2}}{\Gamma^{2}+\left(\Delta E_{\mathrm{F}}+e V_{\mathrm{sd}}\right)^{2}} V_{\mathrm{sd}},
$$

where $\Delta E_{\mathrm{F}}=E_{\mathrm{F}_{2}}-E_{\mathrm{F}_{1}}$, and $G_{0}$ is a constant with the dimensions of conductance. The tunneling linewidth is the sum, $\Gamma=\Gamma_{1}+\Gamma_{2}$, of the widths of the Lorentzian spectral functions in the two layers.14

In this paper we report differential conductance $G=d I / d V_{\text {sd }}$ measurements. The zerobias conductance obtained by differentiating Eq. 2 is

$$
G\left(V_{\mathrm{sd}}=0\right) \propto \int d^{2} \boldsymbol{k} A_{1}\left(\boldsymbol{k}, E_{\mathrm{F}_{1}}\right) A_{2}\left(\boldsymbol{k}, E_{\mathrm{F}_{2}}\right) .
$$

Using Lorentzian spectral functions, the differential conductance as one subband edge (controlled by a gate voltage $V_{\mathrm{g}}$ ) is swept past the other is 14

$$
G\left(V_{\mathrm{g}}\right)=G_{0} \frac{\Gamma^{2}}{\Gamma^{2}+\Delta E_{\mathrm{F}}^{2}} .
$$

Equation 5 predicts Lorentzian $G\left(V_{\mathrm{g}}\right)$ characteristics with a linewidth $\Gamma$ and peaked with magnitude $G_{0}$ at $\Delta E_{\mathrm{F}}=0$, when the carrier densities in the two wells are equal.

We are interested in the differential conductance as a function of $V_{\text {sd }}$, taken when the carrier densities have been matched at $V_{\mathrm{sd}}=0$. However, upon application of an interlayer dc voltage the capacitance between the two layers unavoidably mismatches the carrier densities, and it is incorrect to set $\Delta E_{\mathrm{F}}=0$ in the derivative of Eq. 3 to obtain the conductance

$$
G\left(V_{\mathrm{sd}}\right)=G_{0} \frac{\Gamma^{2}\left(\Gamma^{2}-\left(e V_{\mathrm{sd}}\right)^{2}\right)}{\left(\Gamma^{2}+\left(e V_{\mathrm{sd}}\right)^{2}\right)^{2}} .
$$

There are in fact two contributions to $\Delta E_{\mathrm{F}}$ : One component $\Delta E_{\mathrm{F}}^{0}$ is due to the applied gate voltages (this is zero when the carrier densities are matched at $V_{\mathrm{sd}}=0$ ), and the other, $\Delta E_{\mathrm{F}}^{\mathrm{C}}$, 
is a capacitive effect. $\Delta E_{\mathrm{F}}^{\mathrm{C}}$ is determined by the change of carrier density $\delta n= \pm C V_{\mathrm{sd}} / e$ induced by $V_{\mathrm{sd}}$, where $C=\epsilon / d$ is the capacitance per unit area between the two 2DEGs. The capacitive change in $\Delta E_{\mathrm{F}}$ is $\Delta E_{\mathrm{F}}^{\mathrm{C}}=-2|\delta n| / \rho_{0}=-\beta e V_{\mathrm{sd}}$, where $\rho_{0}=m^{\star} / \pi \hbar^{2}$ is the $2 \mathrm{D}$ density of states, and $\beta=a_{\mathrm{B}} / 2 d$ (where the Bohr radius is $a_{\mathrm{B}} \approx 100 \AA$ in GaAs). Substitution of $\Delta E_{\mathrm{F}}=\Delta E_{\mathrm{F}}^{0}+\Delta E_{\mathrm{F}}^{\mathrm{C}}=\Delta E_{\mathrm{F}}^{0}-\beta e V_{\mathrm{sd}}$ into Eq. 3, followed by differentiation, gives the modified lineshape

$$
G\left(V_{\mathrm{sd}}\right)=G_{0} \frac{\Gamma^{2}\left(\Gamma^{2}+\left(\Delta E_{\mathrm{F}}^{0}\right)^{2}-\left(e V_{\mathrm{sd}}(1-\beta)\right)^{2}\right)}{\left(\Gamma^{2}+\left(\Delta E_{\mathrm{F}}^{0}+e V_{\mathrm{sd}}(1-\beta)\right)^{2}\right)^{2}} .
$$

Setting $\Delta E_{\mathrm{F}}^{0}=0$ (for matched carrier densities at $V_{\mathrm{sd}}=0$ ) gives the lineshape

$$
G\left(V_{\mathrm{sd}}\right)=G_{0} \frac{\Gamma^{2}\left(\Gamma^{2}-\left(e V_{\mathrm{sd}}(1-\beta)\right)^{2}\right)}{\left(\Gamma^{2}+\left(e V_{\mathrm{sd}}(1-\beta)\right)^{2}\right)^{2}},
$$

which has a linewidth of $\Gamma /(1-\beta)$, in contrast to the linewidth $\Gamma$ given in Eq. 6. A typical value of $\beta$ is 0.15 . Therefore, linewidths obtained from $G\left(V_{\mathrm{sd}}\right)$ measurements are approximately $18 \%$ larger than those obtained from equilibrium conductance measurements. 12 Similar capacitive corrections should also be applied to linewidths obtained2 from plots of $I / V_{\text {sd }}$ versus $V_{\text {sd }}$.

We can only quantify capacitive effects at $B=0$, though the magnitude of such effects in a perpendicular magnetic field can be estimated by replacing $\rho_{0}$ with $\rho_{B}$, the density of states at the Fermi level in the outer Landau level. If the outer LL is half-filled, $\rho_{B} \gg \rho_{0}$, then the capacitive change of the Fermi energies $\Delta E_{\mathrm{F}}^{\mathrm{C}}$ will be negligible. The opposite result is obtained near integral filling factors and so we have not made any conclusions from tunneling measurements in this regime.

In Sec. V1 we model high field tunneling measurements obtained at filling factor $\nu=1 / 2$. To model the data we have guessed the form of the spectral density $A_{i}(E)=$ $1 /(2 \pi)^{2} \int d^{2} \boldsymbol{k} A_{i}(\boldsymbol{k}, E)$, and have calculated the tunneling current using the expression

$$
\begin{aligned}
I\left(V_{\mathrm{sd}}\right) \propto & \int d E A_{1}\left(E-E_{0_{1}}\right) A_{2}\left(E-E_{0_{2}}\right) \\
& \times\left[f\left(E-\mu_{1}, T\right)-f\left(E-\mu_{2}, T\right)\right] .
\end{aligned}
$$

\section{TUNNELING IN ZERO MAGNETIC FIELD}

Figure 2 shows the zero-field differential tunneling conductance $G$ as a function of $V_{\mathrm{g}_{1}}$, the front gate voltage; by fixing $V_{\mathrm{g}_{2}}$, the carrier density in the bottom layer was held constant at $n_{2}=3.25 \times 10^{11} \mathrm{~cm}^{-2}$. The equilibrium measurements were taken at $T=3.0 \mathrm{~K}$ (squares) and $19.0 \mathrm{~K}$ (circles). Due to a misalignment between the frontgate and backgate in the tunneling region, there is an unwanted second resonance (which is independent of $V_{\mathrm{g}_{2}}$ ), as well as a small constant background conductance. In Fig. 2 both of these weak contributions

have been removed, and the corrected data at the two temperatures have been fitted to the Lorentzian lineshape

$$
G\left(V_{\mathrm{g}_{1}}\right)=\frac{G_{0}}{1+\left(\frac{V_{\mathrm{g}_{1}}-V_{0}}{\delta V_{\mathrm{g}}}\right)^{2}},
$$


which is Eq. 5 rewritten explicitly in terms of the front gate voltage $V_{\mathrm{g} 1}$. The fits, shown as solid lines, are with $V_{0}=0.046 \mathrm{~V}, \delta V_{\mathrm{g}}=0.0194 \mathrm{~V}$, and $G_{0}=16.1 \mu \mathrm{S}$ at $3 \mathrm{~K}$, and with $V_{0}=$ $0.045 \mathrm{~V}, \delta V_{\mathrm{g}}=0.0360 \mathrm{~V}$, and $G_{0}=9.9 \mu \mathrm{S}$ at $19 \mathrm{~K}$. We have also tried fitting the resonances to a Gaussian and to the derivative of a Fermi function $\left(f^{\prime} \sim \operatorname{sech}^{2}\left[\left(V_{\mathrm{g}_{1}}-V_{0}\right) / \delta V_{\mathrm{g}}\right]\right)$. Of the three functional forms, a Gaussian fit is the poorest, $f^{\prime}$ is considerably better, but the overall shape and the tails of the resonance are best fit by a Lorentzian. The half width at half maximum of the low-temperature Lorentzian resonance, $\delta V_{\mathrm{g}} \approx 19 \mathrm{mV}$, corresponds to an energy broadening $\Gamma \approx 0.5 \mathrm{meV}$. This linewidth $\Gamma$ is the sum of the widths of the spectral functions in the double-layer system, $\Gamma=\hbar / 2 \tau_{1}+\hbar / 2 \tau_{2}$. We relate the linewidth to the scattering rate, defining the average scattering rate of the double-layer system to be $\tau^{-1}=\left(\tau_{1}^{-1}+\tau_{2}^{-1}\right) / 2=\Gamma / \hbar$.

Non-equilibrium measurements provide an alternative method for determining the linewidth $\Gamma$. Figure 3 shows a comparison of the non-equilibrium $G\left(V_{\text {sd }}\right)$ (squares) and equilibrium $G\left(V_{\mathrm{g}_{1}}\right)$ characteristics (circles, and offset vertically) for the same $n_{2}$ for sample C751. To demonstrate the validity of Eq. 7, the non-equilibrium data was obtained with a small mismatch of carrier densities, causing a slight shift of the central $G\left(V_{\mathrm{sd}}\right)$ peak to the left of $V_{\mathrm{sd}}=0$. The solid line through the $G\left(V_{\mathrm{sd}}\right)$ data is a fit to Eq. 7 with $\Delta E_{\mathrm{F}}^{0}=0.08 \mathrm{meV}$, corresponding to a carrier density mismatch of $0.7 \%$. Though small, this mismatch is enough to cause a noticeable asymmetry in the $G\left(V_{\text {sd }}\right)$ characteristics. The fits to both the equilibrium and non-equilibrium traces in Fig. 3 give $\Gamma=0.384 \mathrm{meV}$ and $G_{0}=3.03 \mu \mathrm{S}$. The fitted value $\beta=0.14 \pm 0.01$ is obtained from the non-equilibrium trace and corresponds to an interlayer separation of $d=365 \pm 30 \AA$, somewhat larger than the quantum well centerto-center separation of $305 \AA$ deduced from the growth parameters. However, both parallel magnetic field measurements (using the method of Ref. 17) and self-consistent calculations suggest a center-to-center wavefunction separation of $d \approx 340-350 \AA$.

The successful fits to both the equilibrium and non-equilibrium resonances in Fig. 3 provide strong evidence for a Lorentzian spectral function in zero magnetic field. We have been able to fit Lorentzian lineshapes to the equilibrium tunneling resonances obtained over a wide range of carrier densities, $0.3 \leq n \leq 3.2 \times 10^{11} \mathrm{~cm}^{-2}$, at temperatures from $3 \mathrm{~K}$ to $19 \mathrm{~K}$. From the $n$ and $T$ dependence of the linewidth $\Gamma$, we have investigated the electronimpurity, electron-phonon, and electron-electron contributions to the overall scattering rate

$$
\Gamma / \hbar=\tau^{-1}=\tau_{\mathrm{e}-\mathrm{imp}}^{-1}+\tau_{\mathrm{e}-\mathrm{ph}}^{-1}+\tau_{\mathrm{e}-\mathrm{e}}^{-1}
$$

Figure 1 shows the temperature dependence of the equilibrium linewidth $\Gamma$ at matched carrier densities of $n=0.91,1.62,2.19,3.04 \times 10^{11} \mathrm{~cm}^{-2}$. The solid lines are fits of the form $\Gamma=a+b T^{2}$; at high $n$ the fits are good, but at lower densities the measured linewidth departs from quadratic behavior. The fitting parameters are both functions of $n$, with typical values $a \approx 0.5-0.8 \mathrm{meV}$ and $b \sim 5 \times 10^{-3} \mathrm{meV} / \mathrm{K}^{2}$. Only the electron-phonon and electron-electron scattering terms in Eq. 11 are expected to show significant temperature dependence. In the temperature range considered here, the sheet resistivity $\rho_{x x}$ of each 2DEG shows only a weak temperature dependence, indicating that the mobility is dominated by impurity scattering, and that, by comparison, the temperature dependent electron-phonon contribution is small. We therefore associate the temperature dependent component of the tunneling linewidth with that due to electron-electron scattering $\Gamma_{\mathrm{e}-\mathrm{e}}$. The electron-electron scattering rate in a clean $2 \mathrm{D}$ metal is given by the well-known theoretical expression 18 


$$
\frac{\hbar}{\tau_{\mathrm{e}-\mathrm{e}}}=\frac{E_{\mathrm{F}}}{2 \pi}\left(\frac{k_{\mathrm{B}} T}{E_{\mathrm{F}}}\right)^{2}\left[\ln \left(\frac{E_{\mathrm{F}}}{k_{\mathrm{B}} T}\right)+\ln \left(\frac{2 q_{\mathrm{TF}}}{k_{\mathrm{F}}}\right)+1\right],
$$

where $q_{\mathrm{TF}}=2 / a_{\mathrm{B}}$ is the $2 \mathrm{D}$ Thomas-Fermi screening wavevector. At fixed carrier density $\hbar / \tau_{\text {e-e }}$ exhibits an approximate $T^{2}$ temperature dependence, in agreement with the solid line fits used in Fig. 1.

At low temperatures, the electron-electron scattering is negligible, and $\tau^{-1}$ is dominated by electron-impurity scattering. Figure 5 shows a log-log plot of the equilibrium linewidth versus carrier density at $T=3 \mathrm{~K}$. The fitted gradient is $x=-0.47 \pm 0.05$, which is approximately the power-law behavior expected 19 for small-angle scattering from remote ionized impurities,

$$
\frac{\hbar}{\tau_{\text {e-imp }}}=\frac{\hbar^{2} n_{\mathrm{D}}}{2 m^{\star} s} \sqrt{\frac{\pi}{2}} n^{-1 / 2},
$$

where $n_{\mathrm{D}}$ is the sheet density of impurities, and $s$ is the spacer layer thickness. Equation 13 is valid for $4 k_{\mathrm{F}} s \gg 1$, where $k_{\mathrm{F}}$ is the Fermi wavevector. In our samples the ionized donors are distributed over distances of 400-700 $\AA$ from the center of each quantum well. Taking the smallest distance, $s=400 \AA$, the condition $4 k_{F} s \gg 1$ is easily satisfied for $n=3 \times 10^{11} \mathrm{~cm}^{-2}$, though the applicability of Eq. 13 becomes questionable at the lowest carrier densities.

From experiments over a range of carrier densities and temperatures we have fitted over 300 measured equilibrium linewidths $\Gamma(n, T)$ to the expression

$$
\Gamma(n, T)=\Gamma_{\text {e-imp }}+\Gamma_{\text {e-e }}=a_{1} n^{-0.5}+a_{2} \times[\text { Eq. 12] } .
$$

If $\Gamma$ is measured in units of meV, $n$ in units of $10^{11} \mathrm{~cm}^{-2}$, and $T$ in $\mathrm{K}$, the best fit was obtained with the numerical values $a_{1}=0.77 \pm 0.01$ and $a_{2}=3.06 \pm 0.09$. The dashed lines in Fig. 1 are obtained from this best fit.

The magnitude of the electron-impurity scattering rate, $\Gamma_{\mathrm{e}-\mathrm{imp}}=a_{1} n^{-0.5}$ in Eq. 14, is in agreement with other experiments, 201 but is fifteen times smaller than the theoretical prediction of Eq. 13. Discrepancies between theory and experiment have previously been resolved 22 by including correlations between scatterers. In the high $n$, low $T$ regime, the ratio $\tau^{-1} / \tau_{\mathrm{t}}^{-1} \approx 7$ (where $\tau_{\mathrm{t}}$ is the mobility lifetime) agrees with measurements 20 of similar mobility single-layer GaAs/AlGaAs heterostructures. Furthermore, the scattering time $\tau \approx$ $1.2 \mathrm{ps}$ is comparable to the Dingle time $1.1 \mathrm{ps}$ (obtained from Shubnikov-de Haas oscillations measured on a Hall bar made from the same wafer), confirming the importance of small-angle scattering in determining the tunneling linewidth.

To look at just the electron-electron scattering term, Fig. 6 shows $\Gamma_{\mathrm{e}-\mathrm{e}} / E_{\mathrm{F}}$ plotted versus $k_{\mathrm{B}} T / E_{\mathrm{F}}$. This representation of the data is expected to be nearly universal, 2 though there is some dependence on carrier density inside the square bracket of Eq. 12. This weak dependence on $n$ is illustrated by the two solid line traces of $3.06 \times[\mathrm{Eq}$. 12] (our best fit for $\Gamma_{\text {e-e }}$ ) for $n=0.3$ and $3 \times 10^{11} \mathrm{~cm}^{-2}$ (upper and lower solid curves, respectively). Equation 12 is plotted for $n=1.3 \times 10^{11} \mathrm{~cm}^{-2}$ and without any prefactor $\left(a_{2}=1\right)$ as the dashed line labelled GQ in Fig. 6. Since the original derivation by Giuliani and Quinn 18 there has been some confusion about the value of $a_{2}$ that multiplies this expression. Fukuyama and Abrahams 23 calculated the expression 


$$
\frac{\hbar}{\tau_{\mathrm{e}-\mathrm{e}}}=\pi^{2} \frac{E_{\mathrm{F}}}{2 \pi}\left(\frac{k_{\mathrm{B}} T}{E_{\mathrm{F}}}\right)^{2} \ln \left(\frac{E_{\mathrm{F}}}{k_{\mathrm{B}} T}\right),
$$

and this is shown in Fig. 6 as the curve labelled FA.24 More recent theories2526 highlight the mathematical errors in Ref. 18 and suggest that due to the nature of the tunneling experiment, there are both electron and hole contributions to the linewidth. These two considerations result in $a_{2}=\pi^{2} / 2=4.9$, giving the third dashed line in Fig. 6 labelled JM. Without any energy dependent corrections the various predictions (GQ, FA, and JM) can be compared to our equilibrium measurement $a_{2}=3.06$. The difference between the predictions of GQ and JM and our fit is purely in the value of the prefactor $a_{2}$; whereas the curve FA has a functional form which does not describe the data very well.

The greatest discrepancies between theory and experiment in Fig. 6 occur for large $k_{\mathrm{B}} T / E_{\mathrm{F}}$, for which there are various possible reasons. First, it is assumed in the derivation 18 of Eq. 12 that $E_{\mathrm{F}} \gg k_{\mathrm{B}} T$, a condition which breaks down at low densities and high temperatures. Second, when the matched carrier densities are reduced there is an increase in the disorder of the two layers, and the 2DEGs may no longer be considered as clean 2D metals. The increased level of disorder may also render the Born approximation invalid, in which case the spectral functions and the tunneling resonance are no longer Lorentzian. Third, the derivation of Lorentzian $G\left(V_{\mathrm{g}_{1}}\right)$ characteristics (Eq. 10) from Lorentzian spectral functions is only valid when $\Gamma \ll E_{\mathrm{F}}$; the two energies become comparable when $n=0.5 \times 10^{11} \mathrm{~cm}^{-2}$. The fourth reason is experimental: the pinch-off characteristics of the gate $\left(V_{\mathrm{g}_{1}}\right)$ distort the symmetric equilibrium lineshape, increasing the uncertainty of measured linewidths at low $n$.

In our analysis of the temperature dependence of the equilibrium linewidth we have followed an approach similar to that of Murphy et al., 0 where the non-equilibrium linewidths were measured from $I / V_{\text {sd }}$ versus $V_{\text {sd }}$ characteristics of double 2DEG structures of higher mobility $\left(\mu \approx 10^{6} \mathrm{~cm}^{2} / \mathrm{Vs}\right)$. In our samples the measured electron-electron scattering rate is enhanced over Eq. 12 by a factor $a_{2} \approx 3$, which is two times smaller than that measured in Ref. 2. The two measurements are different, and non-equilibrium linewidths are expected to be larger than equilibrium linewidths for two reasons: an $18 \%$ enhancement can be attributed to charge transfer effects (see Sec. III), and a further 15\% (Ref. 25) to $40 \%$ (Ref. 26) enhancement may be introduced by the energy dependence of $\Gamma_{\text {e-e. }}$. In order to achieve an enhancement factor $a_{2} \approx 6$ close to that of Murphy et al. have concentrated on the energy dependence of $\Gamma_{\mathrm{e}-\mathrm{e}}$, but have neglected the interlayer charge transfer effects. In Fig. 3 we show that, in our samples, we can obtain good agreement between equilibrium and non-equilibrium linewidths by including only charge transfer effects. Moreover, in further investigations over a range of matched and mismatched carrier densities, we find that the energy dependent corrections are consistently small.

There are two major differences between our samples and those of Ref. 2 that might account for the different measured values of $a_{2}$. The first is the level of disorder; in our samples the electron-impurity contribution to the linewidth is $\Gamma_{\text {e-imp }} \approx 0.5 \mathrm{meV}$ compared to $0.25 \mathrm{meV}$ in Ref. 叉. This greater disorder probably does not affect the applicability of Eq. 12, but will make the energy dependent corrections to $\Gamma_{\mathrm{e}-\mathrm{e}}$ less important.27 The second difference is the thickness of the tunnel barrier, which will affect the strength of the interlayer electron-electron interactions. For samples with quantum well widths of $200 \AA$ and barrier thicknesses in the range 175-340 $\AA$ (giving $d=375-540 \AA$ ), Murphy et al. 
measure an enhancement $a_{2}=6.3$ which appears to be independent of barrier thickness. In our samples the interlayer electron-electron distance $(d \approx 340 \AA)$ is comparable to the lowest used in Ref. 2. This could suggest that it is the greater disorder in our samples that gives rise to the smaller enhancement for $\Gamma_{\mathrm{e}-\mathrm{e}}$. By way of comparison, we point out that Berk et al.8 have measured electron-electron scattering rates similar to our own (in the units used here, $a_{2}=3$ ), in 2DEGs with a similarly low mobility $\mu \approx 50-400 \times 10^{3} \mathrm{~cm}^{2} / \mathrm{Vs}$ but more strongly coupled $(d=180 \AA)$. The effect of stronger tunneling (as quantified by the symmetric-antisymmetric gap $\left.\Delta_{\mathrm{SAS}}\right)$ on $\Gamma_{\mathrm{e}-\mathrm{e}}$ has been calculated by Slutzky et al.29

\section{TUNNELING IN A PERPENDICULAR MAGNETIC FIELD}

The application of a perpendicular magnetic field $B$ has a marked effect on the resonant tunneling properties between two 2DEGs. Figure 7 shows the equilibrium conductance $G\left(V_{\text {sd }}=0\right)$, at a matched carrier density of $n=1.0 \times 10^{11} \mathrm{~cm}^{-2}$, as a function of magnetic field at $0.1 \mathrm{~K}$ (solid line) and $1.3 \mathrm{~K}$ (dashed line). As $B$ is increased, the oscillations in the tunneling conductance mirror the formation of LLs in both 2DEGs. However, in contrast to $\mathrm{SdH}$ oscillations, which are an in-plane transport effect, the oscillations shown in Fig. 7 originate from the current flowing perpendicular to the 2DEGs. 30 The oscillations in $G\left(V_{\mathrm{sd}}=0\right)$ can be interpreted in terms of variations of $\rho_{B}$, the DOS at the Fermi level in a field $B$. We will describe the temperature dependence of the equilibrium tunneling in two magnetic field regimes, $B<0.6 \mathrm{~T}$ and $B>0.6 \mathrm{~T}$.

Below 0.6 T, the formation of LLs modulates the DOS, creating minima (maxima) at integral (half-integral) filling factors. The equilibrium tunneling amplitude depends on the DOS in both layers and shows quantum oscillations commensurate with $\rho_{B}$. When the temperature is increased from 0.1 to $1.3 \mathrm{~K}$, the low field tunneling conductance follows the behavior of $\rho_{B}$; the amplitude of the conductance oscillations decrease as the modulation in $\rho_{B}$ becomes weaker, and $G\left(V_{\text {sd }}=0\right)$ tends towards its zero-field value.

For $B<0.6 \mathrm{~T}$ the magnitude of the conductance maxima increase with magnetic field, following the expected increase in the degeneracy of a half-filled LL. However, for $B>0.6 \mathrm{~T}$ the amplitude of the tunneling maxima decrease with increasing magnetic field; this can be interpreted as a magnetic-field-induced suppression of the equilibrium tunneling between the two 2DEGs, with the formation of a gap in the tunneling DOS. When the temperature is raised to $1.3 \mathrm{~K}$ the suppression of the conductance is reduced, indicating thermal activation across the gap. The Fig. 7 inset shows low-field equilibrium tunneling for a matched carrier density of $n=3 \times 10^{11} \mathrm{~cm}^{-2}$. At this higher carrier density the crossover from SdH-like to activated behavior again occurs at $B=0.6 \mathrm{~T}$. These measurements at different temperatures show that the signature of the magnetic-field-induced gap is evident at filling factors as high as $\nu \approx 18$. Clearly, above $0.6 \mathrm{~T}$ a mechanism other than the simple formation of LLs is needed to explain the unusual temperature dependence observed at half-integral filling factors.

The formation of a magnetic-field-induced gap can also be investigated by nonequilibrium measurements, where at low magnetic fields a dip around zero bias is observed in the $G\left(V_{\mathrm{sd}}\right)$ characteristics. Figure 8 shows $G\left(V_{\mathrm{sd}}\right)$ characteristics at various magnetic fields for a matched carrier density of $n=0.95 \times 10^{11} \mathrm{~cm}^{-2}$. In the trace at $B=0.4 \mathrm{~T}$ there is a slight dip at $V_{\mathrm{sd}}=0$; with increasing magnetic field both the depth of the dip and 
the separation of the surrounding peaks become larger. To characterize the tunneling gap, we define $\Delta$ to be the voltage separation of the conductance peaks either side of $V_{\text {sd }}=0$. Previously, we have measured $I\left(V_{\mathrm{sd}}\right)$ characteristics for $\nu<1$ and have successfully fitted our low voltage data to the expression $I=I_{0} \exp \left(-\Delta / e V_{\mathrm{sd}}\right)$. At high fields the values of $\Delta$ obtained from such fits are precisely the same as those measured from the peak separations.8 Although at low magnetic fields we cannot fit our data to this theoretical expression for $I$, we nevertheless use the peak separation as a straightforward measure of the gap. To test various theoretical predictions we have investigated $\Delta$ at fixed $n$ and fixed $\nu$, both as a function magnetic field $B$.

Figure 9 shows the gap parameter $\Delta$, measured from traces similar to those in Fig. 8 , over a wider range of $B$. Due to the presence of edge states we disregard measurements close to the integer quantum Hall $(\mathrm{QH})$ regime, as well as those near the fractional QH state at $\nu=2 / 3$. The linear fit through the remaining points (solid circles) is $\Delta=(0.45 \pm 0.02) \hbar \omega_{\mathrm{c}}-$ $(0.19 \pm 0.05) \mathrm{meV}$. Figure 9 shows that the gap is most cleanly observed at half-integral filling factors, and in measurements at fixed $n$ there are more data points at low $\nu$ than at high $\nu$. With this proviso the linear fit in Fig. 9 spans the whole field regime, though it is difficult to identify deviations at low $B$. We have also performed conductance measurements at a higher matched carrier density of $n=1.48 \times 10^{11} \mathrm{~cm}^{-2}$, and Fig. 10 shows the evolution of the the low field tunneling characteristics at this carrier density presented as a grey-scale plot. The figure is made up of $G\left(V_{\text {sd }}\right)$ characteristics, similar to those in Fig. 8, taken at 101 magnetic fields from $B=0$ to $2 \mathrm{~T}$. To accentuate the data each trace has been scaled between 0 (black) and 1 (white). The light structures around the center, marked with solid lines, are the two tunneling peaks surrounding the gap, with a splitting that is linear in magnetic field, $\Delta=(0.43 \pm 0.03) \hbar \omega_{\mathrm{c}}-(0.26 \pm 0.03) \mathrm{meV}$. Within experimental error the gaps measured at the two carrier densities, $0.95 \times 10^{11} \mathrm{~cm}^{-2}$ and $1.48 \times 10^{11} \mathrm{~cm}^{-2}$, have the same magnetic field dependence.

To obtain more information about the gap at higher filling factors, Fig. 11] shows measurements of $\Delta_{\nu}$ taken as a function of $B$ while maintaining a half-integral filling factor in the tunneling region. Gap measurements were obtained at $\nu=1 / 2,3 / 2, \ldots, 11 / 2$ for seventeen matched carrier densities in the range $0.36 \leq n \leq 3.10 \times 10^{11} \mathrm{~cm}^{-2}$. At a given magnetic field, the higher the half-integral filling factor, the smaller the gap. The DOS is not clearly spin-split below $B \approx 1 \mathrm{~T}$, and so data points for the highest filling factor and lowest fields are subject to some error. The straight line $0.45 \hbar \omega_{\mathrm{c}}-0.19 \mathrm{meV}$ is included in Fig. 11 to demonstrate the linear $B$ dependence of the tunneling gap at $\nu=1 / 2$, and the sublinear nature of the gap at higher filling factors. At low carrier densities $n<1.5 \times 10^{11} \mathrm{~cm}^{-2}$, measurements of $\Delta_{\nu \geq 3 / 2}$ converge to a value similar to that measured at $\nu=1 / 2$. For the higher carrier concentrations the gap for $\nu>3 / 2$ is reduced in comparison with $\Delta_{1 / 2}$. This observation, plus the scarcity of data points at higher filling factors in measurements at fixed $n$ accounts for the same measured gap at $0.95 \times 10^{11} \mathrm{~cm}^{-2}$ and $1.48 \times 10^{11} \mathrm{~cm}^{-2}$. We stress that, as previously reported, 7 the high field gap shows the same linear behavior both at fixed $n$ and fixed $\nu<1$.

Figures 8 and 10 also show tunneling between LLs of different index. At high magnetic fields, the first inter-LL tunneling transition has been observed] 31 as a peak in the $I\left(V_{\text {sd }}\right)$ characteristics at $e V_{\mathrm{sd}} \approx 1.3 \hbar \omega_{\mathrm{c}}$, and the equivalent conductance measurement 8 exhibits a peak at $e V_{\text {sd }} \approx 1.16 \hbar \omega_{\mathrm{c}}$. Both measurements show inter-LL transitions occuring at higher 
than expected bias voltages, and it is proposed 31 that this enhanced LL spacing is due to an effective mass reduced by many-body effects 32 The arrows in Fig. 8 and the dashed lines superimposed on Fig. 10 show the position of the first inter-LL tunneling peaks either side of zero bias. Over the range $0.5 \leq B \leq 3 \mathrm{~T}$ the conductance peaks occur at $e V_{\text {sd }}=(1.07 \pm$ $0.03) \hbar \omega_{c}$, an enhancement that is only slightly less than that observed at higher magnetic fields. If the enhanced LL spacing is due to a reduced effective mass, our measurements suggest that the mass is reduced at higher filling factors by an amount much greater than that predicted by Smith et al.32

\section{Comparison with Theory}

There has been much interest in tunneling in a weak magnetic field where, even for filling factors $\nu \gg 1$, it is believed that there is an energy cost associated with the injection and extraction of electrons into and out of a 2DEG. It has been theoretically shown 33 that at low magnetic fields the bare Coulomb interaction (which typically exceeds the cyclotron energy $\left.\hbar \omega_{\mathrm{c}}\right)$ is screened by the filled LLs, and the renormalized interaction is sufficiently weak that it does not mix LLs. Therefore only interactions between electrons in the outer partially filled LL need to be considered; this is the starting point for many of the theories in the weak field regime.

Following the treatment at high magnetic fields, the gap at lower fields and higher filling factors can be considered to be the Coulomb energy, where the cyclotron length $l_{\mathrm{c}}=\hbar k_{\mathrm{F}} / e B$ replaces the magnetic length $l_{B}$ as the relevant length scale. The semiclassical energy gap is

$$
\Delta_{1}=\frac{e^{2}}{4 \pi \epsilon l_{\mathrm{c}}}=\frac{r_{\mathrm{s}} \hbar \omega_{\mathrm{c}}}{\sqrt{2}}
$$

where $r_{\mathrm{s}}$ is the interaction parameter, and is given by $r_{\mathrm{s}}=a / a_{\mathrm{B}}=\sqrt{2} / a_{\mathrm{B}} k_{\mathrm{F}}$. Levitov and Shytov 34 consider the problem of an electron orbiting in a ring of thickness $l_{B}$ and radius $l_{c}$ in one layer, tunneling into a similar ring in the second layer, leaving behind a hole in the first ring. The electrostatic energy of this two-ring capacitor creates an energy gap that is proportional to Eq. 16, but multiplied by a logarithmic screening term. Alternatively, using both a hydrodynamic approach 33 and a Hartree-Fock calculation, 35 Aleiner and coworkers have obtained a gap of the form

$$
\Delta_{2}=\frac{\hbar \omega_{\mathrm{c}}}{2 \nu} \ln \left(r_{\mathrm{s}} \nu\right)
$$

It has been shown 34 that the total gap has contributions from two terms similar in form to $\Delta_{1}$ and $\Delta_{2}$, where $\Delta_{1}$ is the dominant term.

In a recent theory, Fogler et al. 36 propose that under certain conditions the ground state of the 2DEG in a weak magnetic field is a charge density wave (CDW) superimposed on a uniform background of filled LLs. Tunneling into a partially filled LL disturbs some of the correlations of the CDW, and the energy cost of this disruption gives rise to a tunneling gap

$$
\Delta_{3}=\frac{r_{\mathrm{s}} \hbar \omega_{\mathrm{c}}}{\sqrt{2} \pi} \ln \left(1+\frac{0.3}{r_{\mathrm{s}}}\right)+\Delta_{2} \approx 0.07 \hbar \omega_{\mathrm{c}}+\Delta_{2}
$$


As $B$ is decreased there is a predicted 36 crossover from the spin-resolved QH regime (spinsplit LLs) where the ground state is a CDW, to the spin-unresolved QH regime (well separated, but spin-degenerate LLs). The CDW ground state can only exist in the former regime, while at lower fields the ground state is a uniform electron liquid which is expected 36 to have a gap of a different origin. In our samples the $\mathrm{SdH}$ oscillations of the individual 2DEGs show that the spin-unresolved QH regime occurs over a narrow range of magnetic field. The Fig. 7 inset shows that at $n=3 \times 10^{11} \mathrm{~cm}^{-2}$ it is possible to observe a suppression of tunneling (above $0.6 \mathrm{~T}$ ) in the absence of spin-splitting (which becomes distinct for $B>1.5 \mathrm{~T}$ ). Moreover, in all our measurements over a wide range of $n$, we see no clear change in the character of the gap once spin-splitting has set in. The $B$ dependence of the low field gaps predicted by Aleiner et al.35 and Fogler et al. 36 are determined by $\Delta_{2}$, which varies as $\Delta_{2} \sim B^{2}$ at fixed carrier density, and as $\Delta_{2} \sim B$ at fixed filling factor. Figure 9 shows that at fixed $n$ the gap is linear (not quadratic) in $B$, and Fig. 11 shows that at fixed $\nu$ the weak field gap $\Delta_{\nu \geq 3 / 2}$ is sublinear in $B$.

The semiclassical gap (Eq. 16) behaves as $\Delta_{1} \sim B$ at fixed carrier concentration, and as $\Delta_{1} \sim \sqrt{B}$ at fixed filling factor. For fixed $n$ the measured gaps are indeed linear in $B$, though we find that the best fits are obtained with a negative intercept. Similarly the best fits of $\Delta_{\nu}$ to a $\sqrt{B}$ field dependence are also obtained with a negative intercept, using the expression $\Delta_{\nu}=a^{\prime} \sqrt{B / \nu}-b^{\prime}$. In the inset to Fig. 11] we have plotted $\left(\Delta_{\nu}+b^{\prime}\right) \nu^{1 / 2}$ versus $B^{1 / 2}$ for $\nu \geq 5 / 2$. Taking $b^{\prime}=0.4 \mathrm{meV}$ the data collapse onto a single straight line with slope $a^{\prime}=2.0$. We conclude from measurements at constant $n$ and constant $\nu$ that the high filling factor gap is best described by

$$
\Delta=(0.46 \pm 0.03) \frac{e^{2}}{4 \pi \epsilon l_{\mathrm{c}}}-(0.4 \pm 0.1) \mathrm{meV}
$$

The negative intercept could be interpreted as evidence for an excitonic effect (similar to that observed 9 at $\nu=1 / 2$ ), and with a magnitude which is approximately independent of filling factor for $\nu \geq 5 / 2$. We were unable to obtain good agreement between Eq. 19 and the measured gaps at $\nu=1 / 2$ and $3 / 2$, instead much larger negative intercepts $\left(b^{\prime}=4.2\right.$ and $1.7 \mathrm{meV}$, respectively) were required. With our samples we have obtained 8 better agreement at $\nu=1 / 2$ using a linear rather square root magnetic field dependence for the gap $\Delta_{1 / 2}$ up to $25 \mathrm{~T}$.

\section{TEMPERATURE STUDIES IN HIGH FIELDS}

In this section we use Eq. 9 to model high field $G\left(V_{\mathrm{sd}}\right)$ measurements taken at $\nu=$ $1 / 2$, results which have been presented elsewhere.37 Figure 12.(a) shows the conductance characteristics at $B=8 \mathrm{~T}$ at a matched carrier density of $n_{1}=n_{2}=0.97 \times 10^{11} \mathrm{~cm}^{-2}$. As the temperature is increased from $T=1.5 \mathrm{~K}$ to $6 \mathrm{~K}$, the equilibrium conductance $G\left(V_{\mathrm{sd}}=0\right)$ increases, while the height of the surrounding peaks is reduced. Figure 12(b) shows $G\left(V_{\text {sd }}=0\right)$ in an Arrhenius plot, revealing activated behavior at low temperatures with an activation energy of $E_{\mathrm{a}}=0.35 \pm 0.01 \mathrm{meV}$. Above the activation temperature $(\approx 4 \mathrm{~K})$ the equilibrium conductance departs from exponential behavior, and for $T>10 \mathrm{~K}$ the conductance decreases. We have measured $E_{\mathrm{a}}$ at different matched carrier densities 
and magnetic fields, while maintaining a filling factor of $\nu=1 / 2$ in the tunneling region. Figure 13 shows $E_{\mathrm{a}}$ as a function of magnetic field, with a fit which shows that the activation energy is proportional to $B$. At $\nu=1 / 2$ the ratio $E_{\mathrm{a}} / \Delta$ is $0.047 \pm 0.005$, and is approximately independent of $B$. Activation plots of the high field gap were first obtained by Eisenstein et al., and for comparison we measure from Fig. 3 of Ref. 31 the ratio $E_{\mathrm{a}} / \Delta=0.07$ at $B=13 \mathrm{~T}$.

Temperature can be used to probe the shape of the tunneling DOS, and previously Ashoori et al. 10 have used a DOS with a linear dip at the Fermi level to model 2D-3D tunneling measurements. A linear DOS does not give activated behavior, and to obtain such characteristics we have modeled the data using the double-Gaussian spectral density

$$
A(E) \propto \frac{1}{\sqrt{E_{0} k_{\mathrm{B}} T}}\left\{\exp \left(-\frac{\left(E_{0}-E\right)^{2}}{2 \alpha E_{0} k_{\mathrm{B}} T}\right)+\exp \left(-\frac{\left(E_{0}+E\right)^{2}}{2 \alpha E_{0} k_{\mathrm{B}} T}\right)\right\},
$$

where the Fermi level $E_{\mathrm{F}}$ lies in the minimum between the two peaks. This tunneling DOS is a modified version of Eq. 14 in Ref. 33, which was originally proposed for $\nu \gg 1$ and $E_{0} \gg k_{\mathrm{B}} T$. By comparison with the original formula, we have introduced an adjustable parameter $\alpha$ in the denominator of the exponential. As $T \rightarrow 0$ the double-Gaussian spectral density consists of two $\delta$-functions at $E= \pm E_{0}$, and so the gap $\Delta \rightarrow 4 E_{0}$ at $T=0$. At low temperatures the activation energy $E_{\mathrm{a}} \rightarrow E_{0} / \alpha$, and so $\Delta$ and $E_{\mathrm{a}}$ share the same magnetic field dependence (that of $E_{0}$ ), in agreement with experiment.

To simulate the conductance measurements in Fig. 12 we have chosen $\alpha=5.3$ so that the theoretical ratio $E_{\mathrm{a}} / \Delta=1 / 4 \alpha$ agrees with the experimental value of $0.047 . \quad I\left(V_{\mathrm{sd}}\right)$ characteristics have been calculated using Eq. 20 in Eq. 9, and by numerical differentiation of these characteristics we obtain the $G\left(V_{\mathrm{sd}}\right)$ traces shown in Fig. 14(a). The value of $E_{0}=2.6 \mathrm{meV}$ best reproduces the conductance and activation characteristics (see Fig. 14(b)) at $8 \mathrm{~T}$. From similar simulations of $\nu=1 / 2$ data at different magnetic fields we have measured the $B$ dependence of $E_{0}$; the results are displayed in Fig. 13, with a fit showing $E_{0}$ with a linear dependence on the magnetic field, $E_{0}=0.2 \hbar \omega_{\mathrm{c}}$.

The calculated $G\left(V_{\text {sd }}\right)$ curves reproduce many features of the experimental data. First, there are bias voltages which delineate regions of positive and negative $\partial G / \partial T$; second, the conductance maxima move closer together with increasing temperature; third, it shows

that at higher temperatures $G\left(V_{\mathrm{sd}}=0\right)$ is no longer activated, but reduces with increasing temperature. There are, however, some differences between the model and experiment. The very low temperature limit of the experimental characteristics differ from those of the model; in the experimental system disorder introduces a finite width to the DOS at $T=0$. We have tried to simulate this disorder by introducing a finite offset temperature, that is by replacing $T$ with $T+T_{0}$ in Eq. 20, where $T_{0}$ is a constant. However, there was little improvement in the shape of the simulated characteristics.

\section{CONCLUSIONS}

We have used both the equilibrium and non-equilibrium tunneling characteristics to show that the underlying spectral function, $A(\boldsymbol{k}, E)$, within each of the 2DEGs of a doublelayer system is a Lorentzian. Equilibrium measurements show that the Lorentzian tunneling linewidth has a carrier density and temperature dependence that can be attributed to 
electron-impurity and electron-electron scattering. The measured electron-impurity scattering rate is similar in magnitude to the Dingle time, and comparison with theory shows that correlations between scatterers need to be taken into account to obtain better agreement with experiment. The mathematical form, but not the magnitude, of the electron-electron scattering rate measured from the equilibrium lineshape is well described by theory.

In a perpendicular magnetic field the equilibrium tunneling conductance oscillates as a function of $B$, reflecting the formation of LLs. For $B>0.6 \mathrm{~T}$ the magnetic field creates a gap in the tunneling DOS which suppresses the equilibrium tunneling. For fixed filling factor $v=1 / 2$ the tunneling gap has a linear magnetic field dependence, whereas at higher half-integral filling factors the gap is sublinear and is best described by a semiclassical gap. We have modeled the temperature dependence of the conductance characteristics in a strong magnetic field using a double-Gaussian spectral density.

\section{ACKNOWLEDGMENTS}

We wish to thank the Engineering and Physical Sciences Research Council (UK) for supporting this work. JTN acknowledges support from the Isaac Newton Trust, and DAR acknowledges support from Toshiba Cambridge Research Centre. We thank A.V. Khaetskii for a critical reading of the manuscript, and we acknowledge help with the numerical calculations from I. M. Castleton and C. H. W. Barnes. 


\section{REFERENCES}

${ }^{1}$ J. Smoliner et al., Phys. Rev. Lett. 63, 2116 (1989).

${ }^{2}$ S. Q. Murphy, J. P. Eisenstein, L. N. Pfeiffer, and K. W. West, Phys. Rev. B 52, 14825 (1995).

${ }^{3}$ S.-R. E. Yang and A. H. MacDonald, Phys. Rev. Lett. 70, 4110 (1993).

${ }^{4}$ Y. Hatsugai, P.-A. Bares, and X. G. Wen, Phys. Rev. Lett. 71, 424 (1993).

${ }^{5}$ S. He, P. M. Platzman, and B. I. Halperin, Phys. Rev. Lett. 71, 777 (1993).

${ }^{6}$ P. Johansson and J. M. Kinaret, Phys. Rev. Lett. 71, 1435 (1993).

${ }^{7}$ K. M. Brown et al., Phys. Rev. B 50, 15465 (1994).

${ }^{8}$ K. M. Brown et al., Physica B 211, 430 (1995).

${ }^{9}$ J. P. Eisenstein, L. N. Pfeiffer, and K. W. West, Phys. Rev. Lett. 74, 1419 (1995).

${ }^{10}$ R. C. Ashoori, J. A. Lebens, N. P. Bigelow, and R. H. Silsbee, Phys. Rev. B 48, 4616 (1993).

${ }^{11}$ R. C. Ashoori, private communication, (1995).

${ }^{12}$ K. M. Brown et al., in Proceedings of the 22nd Conference on the Physics of Semiconductors, Vancouver, Canada, 1994, edited by D. J. Lockwood (World Scientific, Singapore, 1995), p. 1035.

${ }^{13}$ K. M. Brown et al., Appl. Phys. Lett. 64, 1827 (1994).

${ }^{14}$ L. Zheng and A. H. MacDonald, Phys. Rev. B 47, 10619 (1993).

${ }^{15}$ G. D. Mahan, in Many-Particle Physics (Plenum Press, NY, 1993).

${ }^{16}$ E. L. Wolf, in Principles of Electron Tunneling Spectroscopy (Oxford University Press, NY, 1985).

17 J. P. Eisenstein, T. J. Gramila, L. N. Pfeiffer, and K. W. West, Phys. Rev. B 44, 6511 (1991).

${ }^{18}$ G. F. Giuliani and J. J. Quinn, Phys. Rev. B 26, 4421 (1982).

19 A. Gold, Phys. Rev. B 38, 10798 (1988).

${ }^{20}$ P. T. Coleridge, Phys. Rev. B 44, 3793 (1991).

${ }^{21}$ B. Das, S. Subramaniam, M. R. Melloch, and D. C. Miller, Phys. Rev. B 47, 9650 (1993).

${ }^{22}$ P. J. van Hall, Superlatt. Microstruct. 6, 213 (1989).

${ }^{23}$ H. Fukuyama and E. Abrahams, Phys. Rev. B 27, 5976 (1983).

${ }^{24}$ Equation 15 contains only the leading term of Eq. 12, and can be considered2 to have a prefactor $a_{2}=\pi^{2}$.

25 T. Jungwirth and A. H. MacDonald, Phys. Rev. B 53, 7403 (1996).

${ }^{26}$ L. Zheng and S. Das Sarma, Phys. Rev. B 53, 9964 (1996).

${ }^{27}$ L. Zheng, private communication, (1996).

${ }^{28}$ Y. Berk et al., Phys. Rev. B 51, 2604 (1995).

${ }^{29}$ M. Slutzky et al., Phys. Rev. B 53, 4065 (1996).

${ }^{30}$ Although portions of the two 2DEGs close to the Ohmic contacts have carrier densities different to $n_{1}$ and $n_{2}$, the resistance of these leads is always much smaller than the tunneling resistance, and the results presented here depend only on the matched carrier densities in the tunneling region.

31 J. P. Eisenstein, L. N. Pfeiffer, and K. W. West, Phys. Rev. Lett. 69, 3804 (1992).

32 A. P. Smith, A. H. MacDonald, and G. Gumbs, Phys. Rev. B 45, 8829 (1992).

${ }^{33}$ I. L. Aleiner, H. U. Baranger, and L. I. Glazman, Phys. Rev. Lett. 74, 3435 (1995).

${ }^{34}$ L. S. Levitov and A. V. Shytov, preprint cond-mat/9507058 (unpublished). 
${ }^{35}$ I. L. Aleiner and L. I. Glazman, Phys. Rev. B 52, 11296 (1995).

${ }^{36}$ M. M. Fogler, A. A. Koulakov, and B. I. Shklovskii, Phys. Rev. B 54, 1153 (1996).

${ }^{37}$ N. Turner et al., Sol. St. Electron. 40, 413 (1996). 


\section{FIGURES}

FIG. 1. Conduction band profile of the double 2DEG structure, defining the various energies used in Sec. III.

FIG. 2. Equilibrium tunneling conductance $G\left(V_{\mathrm{sd}}=0\right)$ as a function of the gate voltage $V_{\mathrm{g}_{1}}$ controlling the carrier density in the upper layer, when the lower layer density is fixed at $n_{2}=3.25 \times 10^{11} \mathrm{~cm}^{-2}$. The traces were taken at $T=3 \mathrm{~K}$ (squares) and $19 \mathrm{~K}$ (circles), and the solid lines are the fits to Eq. 10 .

FIG. 3. Comparison of non-equilibrium (squares) and equilibrium (circles) tunneling lineshapes at $0.1 \mathrm{~K}$. The solid lines are fits to Eqs. 0 and 10, respectively. For clarity not all the experimental data points are shown, and the equilibrium data and fit have been vertically offset by $1 \mu \mathrm{S}$.

FIG. 4. Temperature dependence of the equilibrium linewidth $\Gamma$ for $n_{1}=n_{2}=0.91,1.62,2.19,3.04 \times 10^{11} \mathrm{~cm}^{-2}$. The solid lines are fits to the form $a+b T^{2}$, and the dashed lines are obtained from the best fit to Eq. 14 .

FIG. 5. Log-log plot of the linewidth $\Gamma$ versus carrier density $n$ at $T=3 \mathrm{~K}$.

FIG. 6. $\Gamma_{\text {e-e }} / E_{\mathrm{F}}$ plotted versus $k_{\mathrm{B}} T / E_{\mathrm{F}}$. The dashed curves are the theoretical predictions of GQ, 18 FA, 23 and JM25 plotted for $n=1.3 \times 10^{11} \mathrm{~cm}^{-2}$. The solid curves are the best fit to Eq. 12 with a prefactor of $a_{2}=3.06$, and with $n=0.3$ and $3 \times 10^{11} \mathrm{~cm}^{-2}$ (upper and lower curves, respectively).

FIG. 7. Main figure: equilibrium tunneling conductance at a matched carrier density of $n=1.0 \times 10^{11} \mathrm{~cm}^{-2}$, taken as a function of perpendicular magnetic field. The solid line shows data taken at $T=0.1 \mathrm{~K}$, and the dashed line shows data taken at $T=1.3 \mathrm{~K}$. Inset: similar measurements taken at $n=3.0 \times 10^{11} \mathrm{~cm}^{-2}$. Filling factors for four of the minima are indicated.

FIG. 8. The conductance characteristics $G\left(V_{\mathrm{sd}}\right)$ in a perpendicular magnetic field at $T=0.1 \mathrm{~K}$, taken at a matched carrier density of $n=0.95 \times 10^{11} \mathrm{~cm}^{-2}$. Sweeps were taken at $B=0,0.4,0.9,2.6 \mathrm{~T}$ with the filling factors indicated. The curves are offset vertically with zeros indicated by the dashed lines. The splitting of the zero field resonance into two peaks defines the gap of width $\Delta$. Arrows indicate the position of inter-Landau level transitions.

FIG. 9. The gap parameter $\Delta$ at matched carrier density $n=0.95 \times 10^{11} \mathrm{~cm}^{-2}$ as a function of magnetic field, measured from traces similar to those shown in Fig. 8. The linear fit, $\Delta=0.45 \hbar \omega_{\mathrm{c}}-0.19 \mathrm{meV}$, disregards measurements (open circles) where the filling factor approaches integral or fractional $(2 / 3)$ values. 
FIG. 10. A grey-scale plot of the tunneling conductance $G$ as a function of $V_{\text {sd }}$ and magnetic field $B$ for $n_{1}=n_{2}=1.48 \times 10^{11} \mathrm{~cm}^{-2}$. Light areas correspond to regions of high conductance. The solid lines show the splitting of the tunneling resonance, with a fitted separation of $\Delta=0.43 \hbar \omega_{\mathrm{c}}-0.26 \mathrm{meV}$. Dashed lines indicate inter-LL transitions at $e V_{\mathrm{sd}}= \pm 1.07 \hbar \omega_{\mathrm{c}}$. The horizontal stripes at $B=1 \mathrm{~T}$ and $1.5 \mathrm{~T}$ show where the tunneling is completely suppressed in the QH regime at filling factors $\nu=6$ and $\nu=4$, respectively.

FIG. 11. Main figure: the gap parameter $\Delta_{\nu}$ at fixed half-integral filling factors $\nu$ as a function of magnetic field. The solid line shows the expression $\Delta_{1 / 2}=0.45 \hbar \omega_{\mathrm{c}}-0.19 \mathrm{meV}$. Inset: $\left(\Delta_{\nu}+0.4 \mathrm{meV}\right) \nu^{1 / 2}$ plotted versus $B^{1 / 2}$ for $\nu \geq 5 / 2$. The slope of the solid line gives $a^{\prime}=2.0$.

FIG. 12. (a) The conductance characteristics $G\left(V_{\mathrm{sd}}\right)$ at $B=8 \mathrm{~T}$ and $\nu=1 / 2$ at temperatures of $T=1.5,1.9,2.4,2.8,3.5,4.3,5.0,6.0 \mathrm{~K}$. (b) Arrhenius plot of $G\left(V_{\mathrm{sd}}=0\right)$ versus $1 / T$ at $B=8 \mathrm{~T}$ and $\nu=1 / 2$. The linear fit at low temperatures gives an activation energy $E_{\mathrm{a}}=0.35 \pm 0.01 \mathrm{meV}$.

FIG. 13. The measured activation energy $E_{\mathrm{a}}$ at $\nu=1 / 2$ (circles) and the best fit value of $E_{0}$ (squares), as a function of magnetic field.

FIG. 14. (a) Conductance curves calculated using the double-Gaussian spectral density (Eq. 20) with $E_{0}=2.6 \mathrm{meV}$ and $\alpha=5.3$. (b) Arrhenius plot of the calculated conductance $G\left(V_{\mathrm{sd}}=0\right)$, showing an activation energy of $0.35 \mathrm{meV}$. 


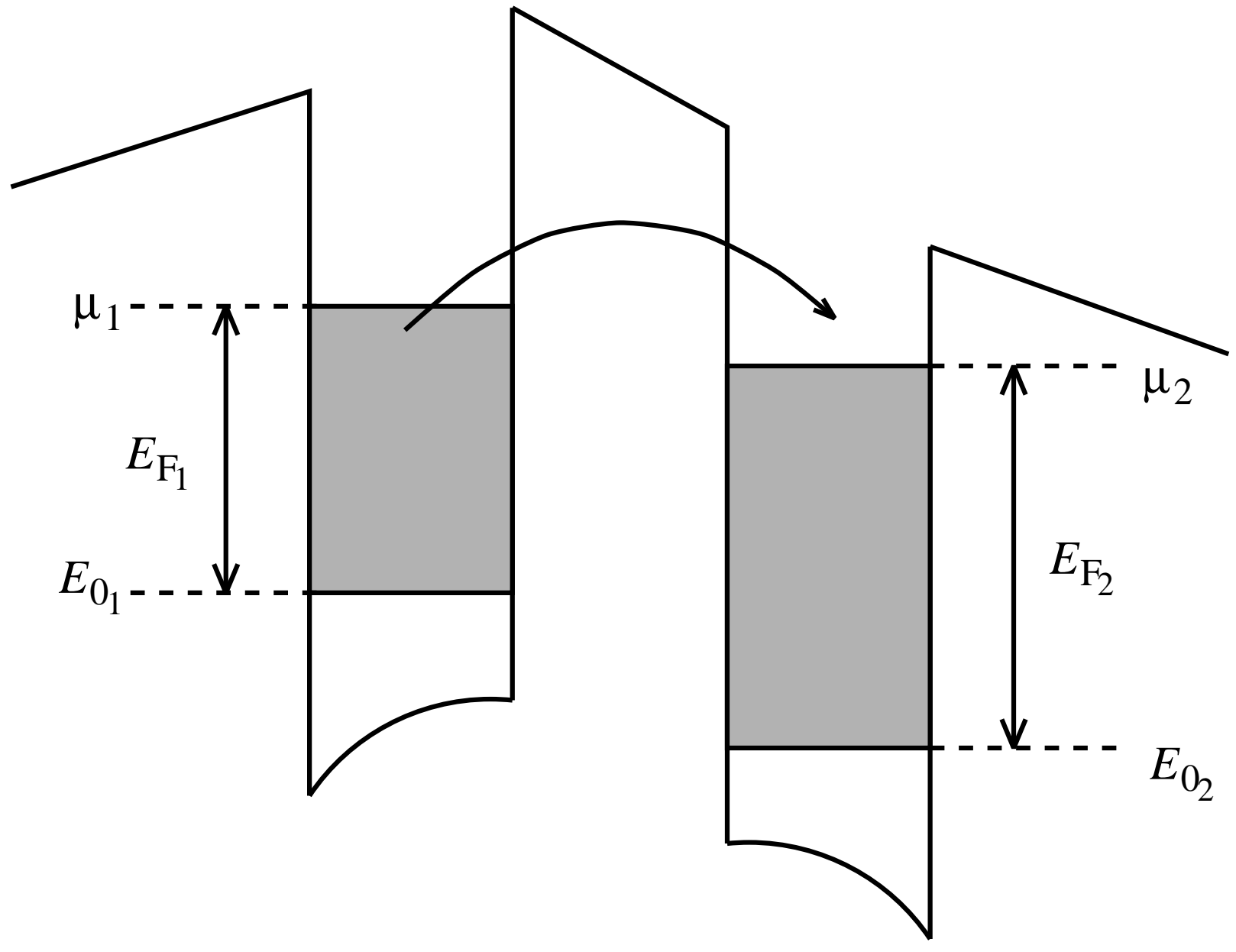




\section{FIG 2}

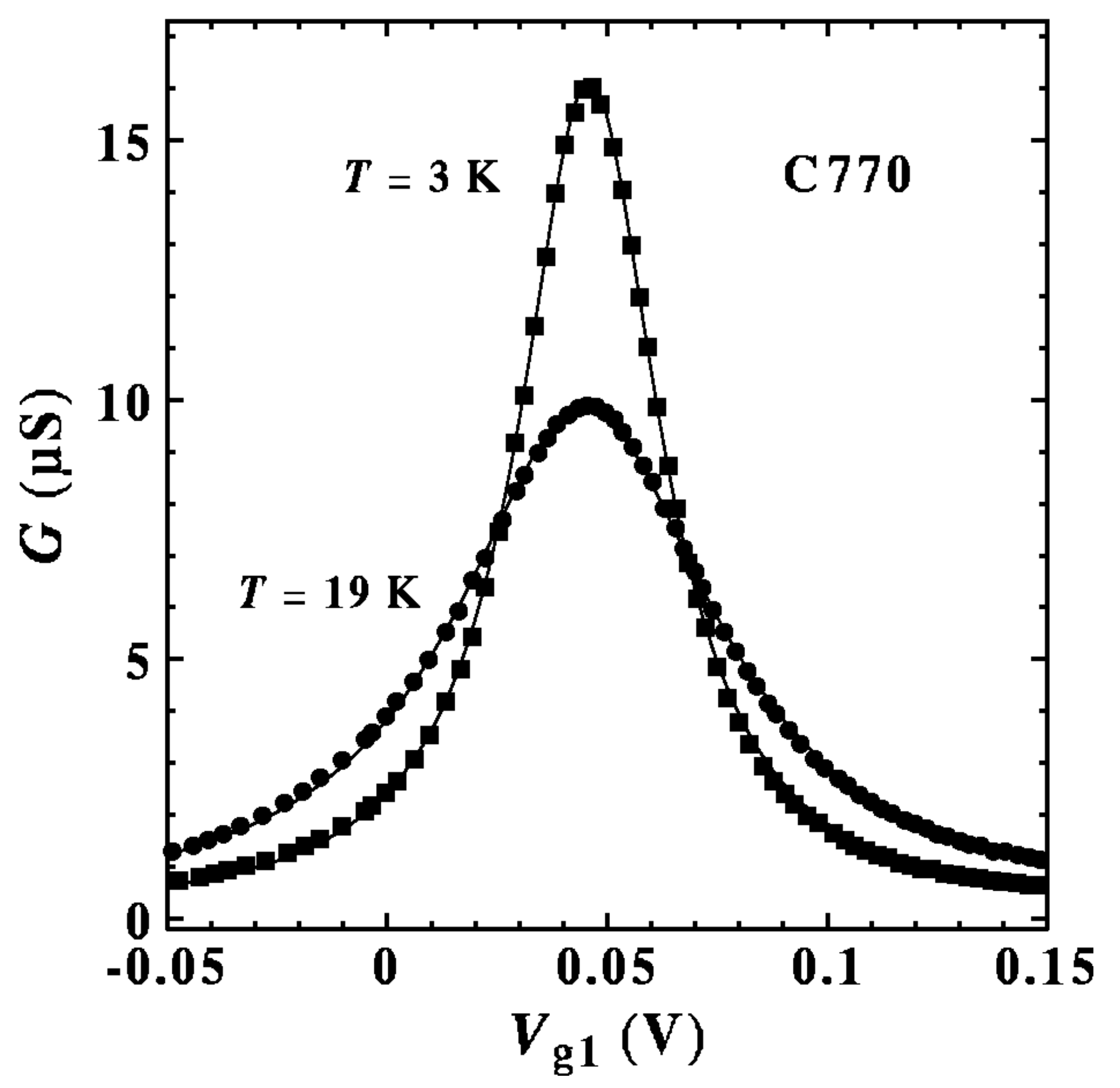




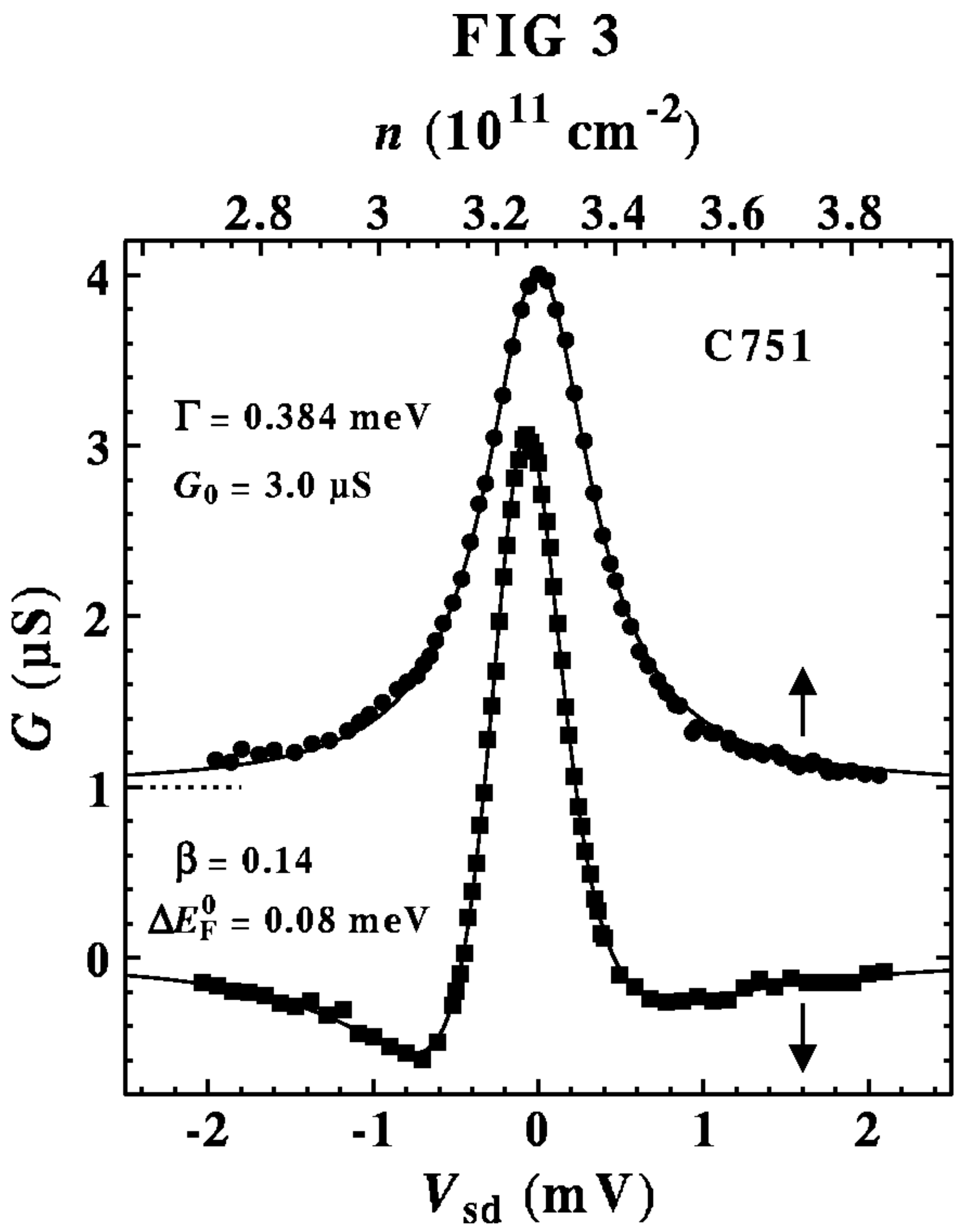




\section{FIG 4}

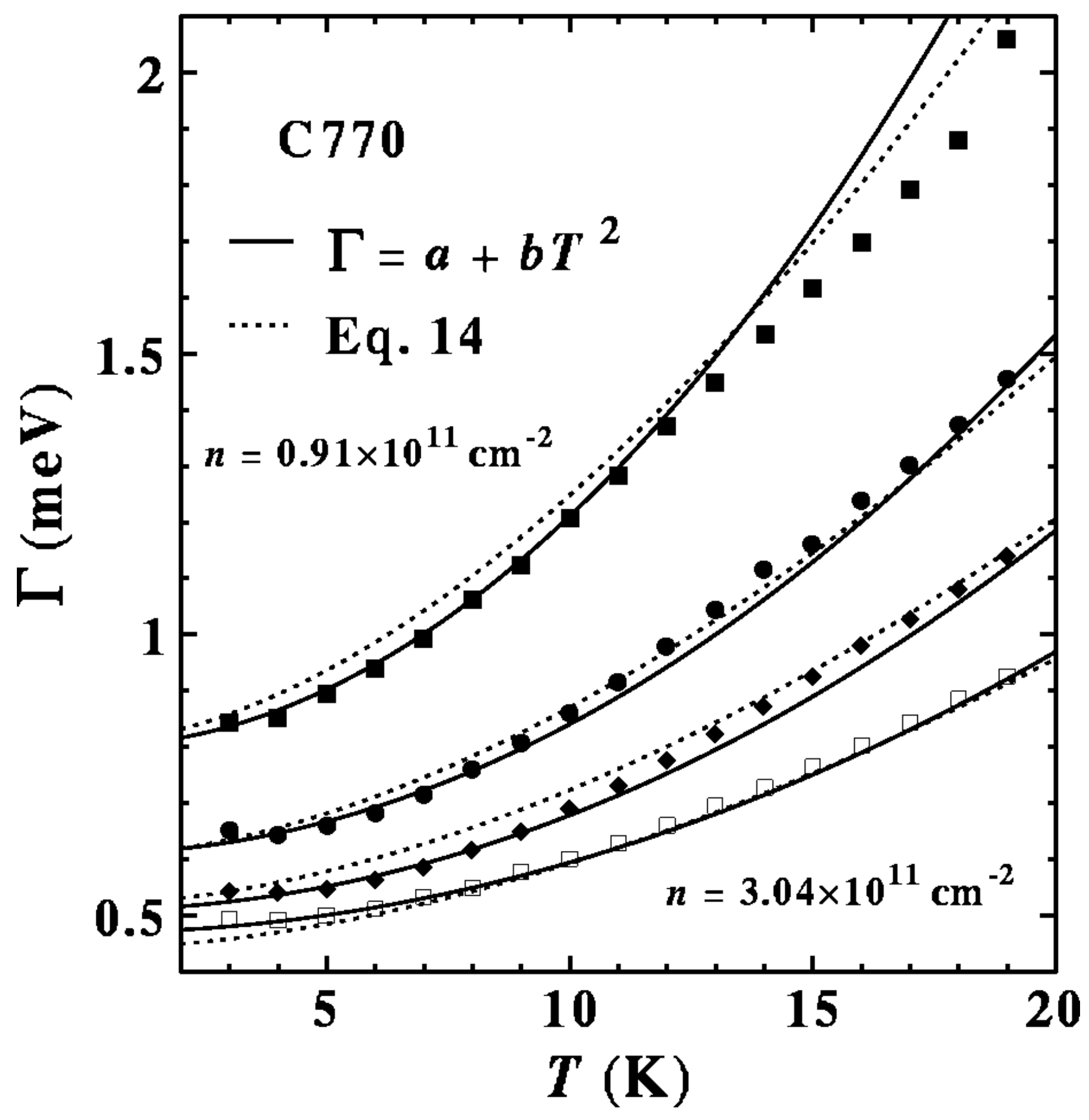




\section{FIG 5}

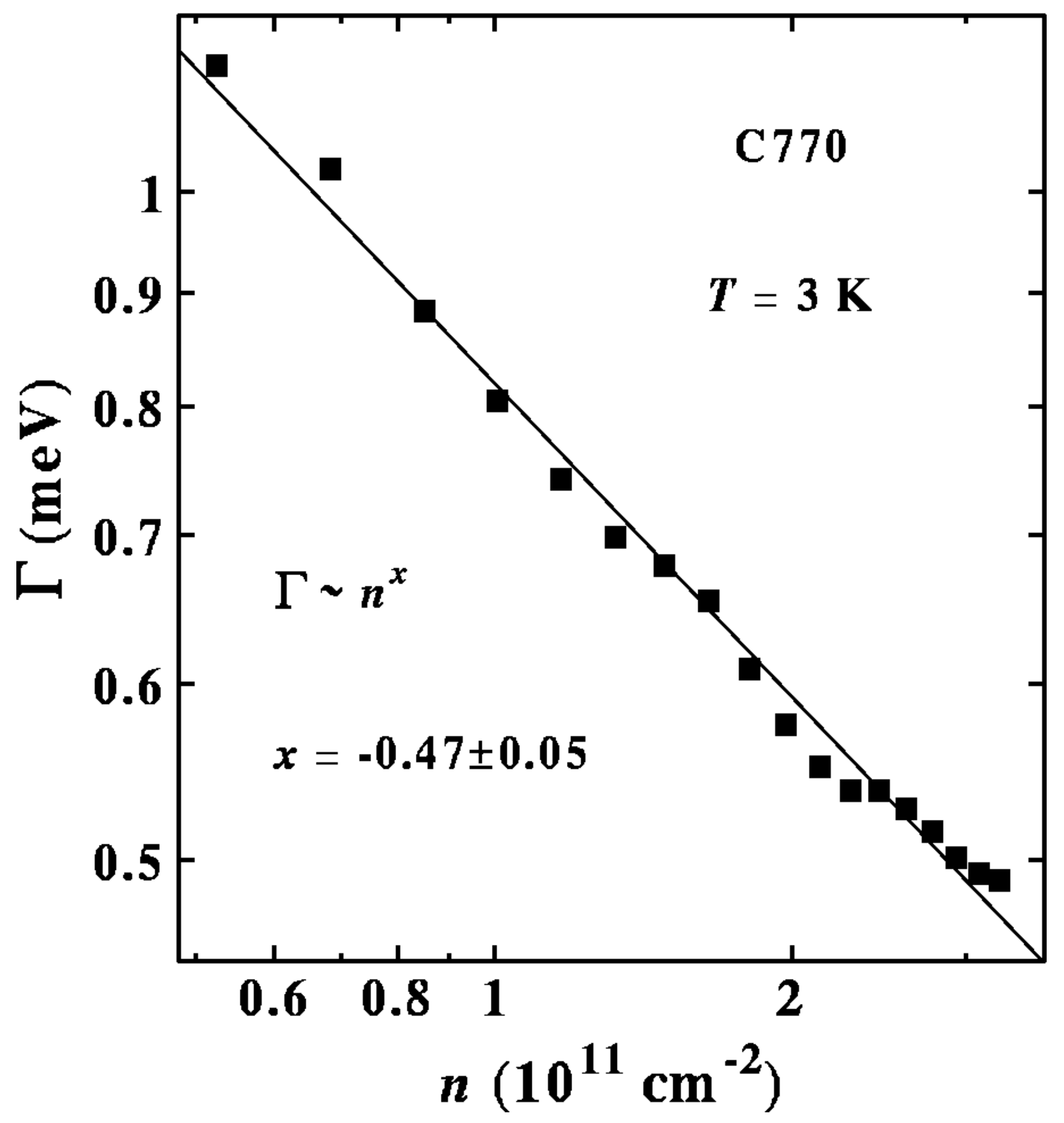




\section{FIG 6}

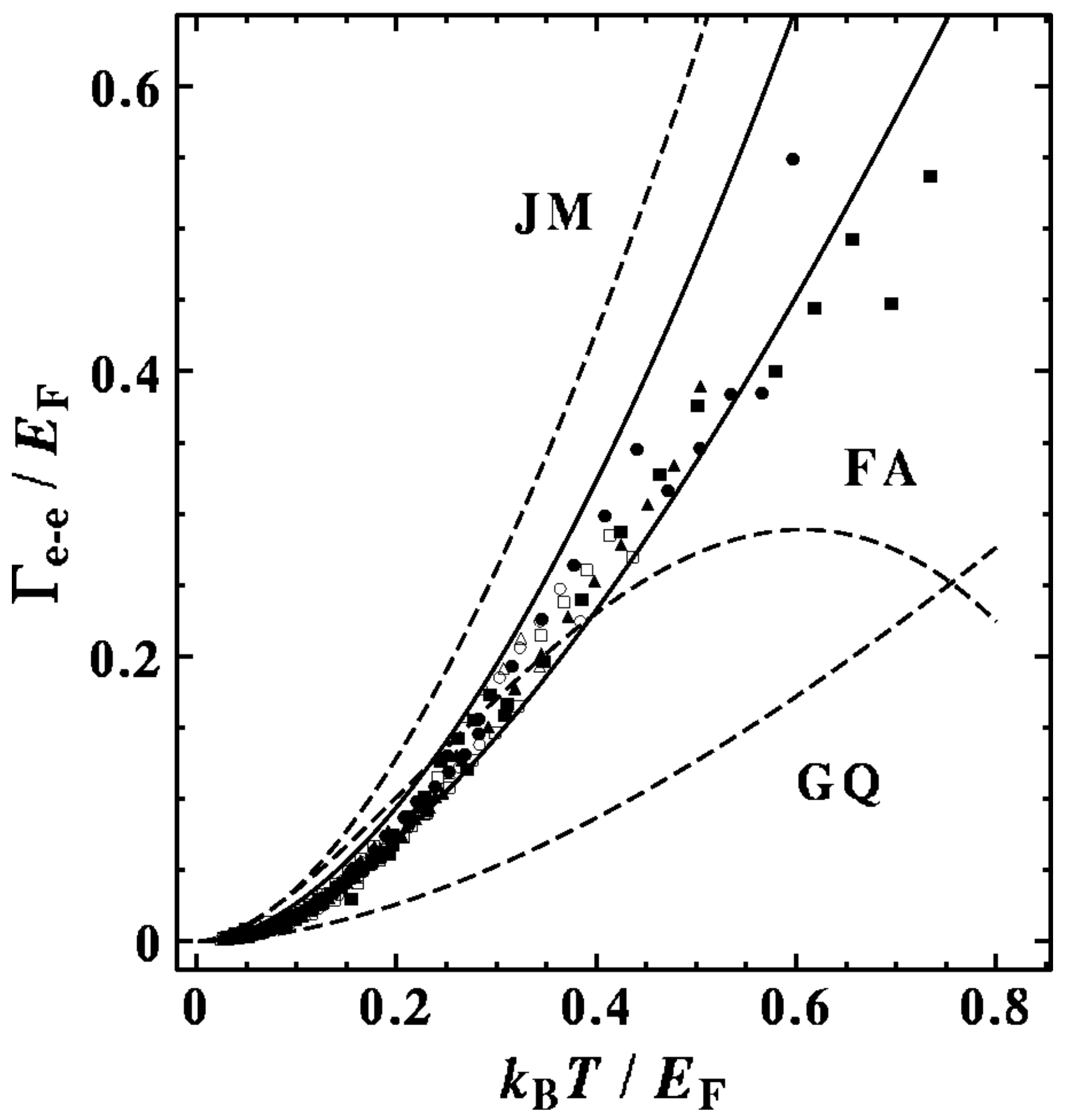




\section{F IG 7}

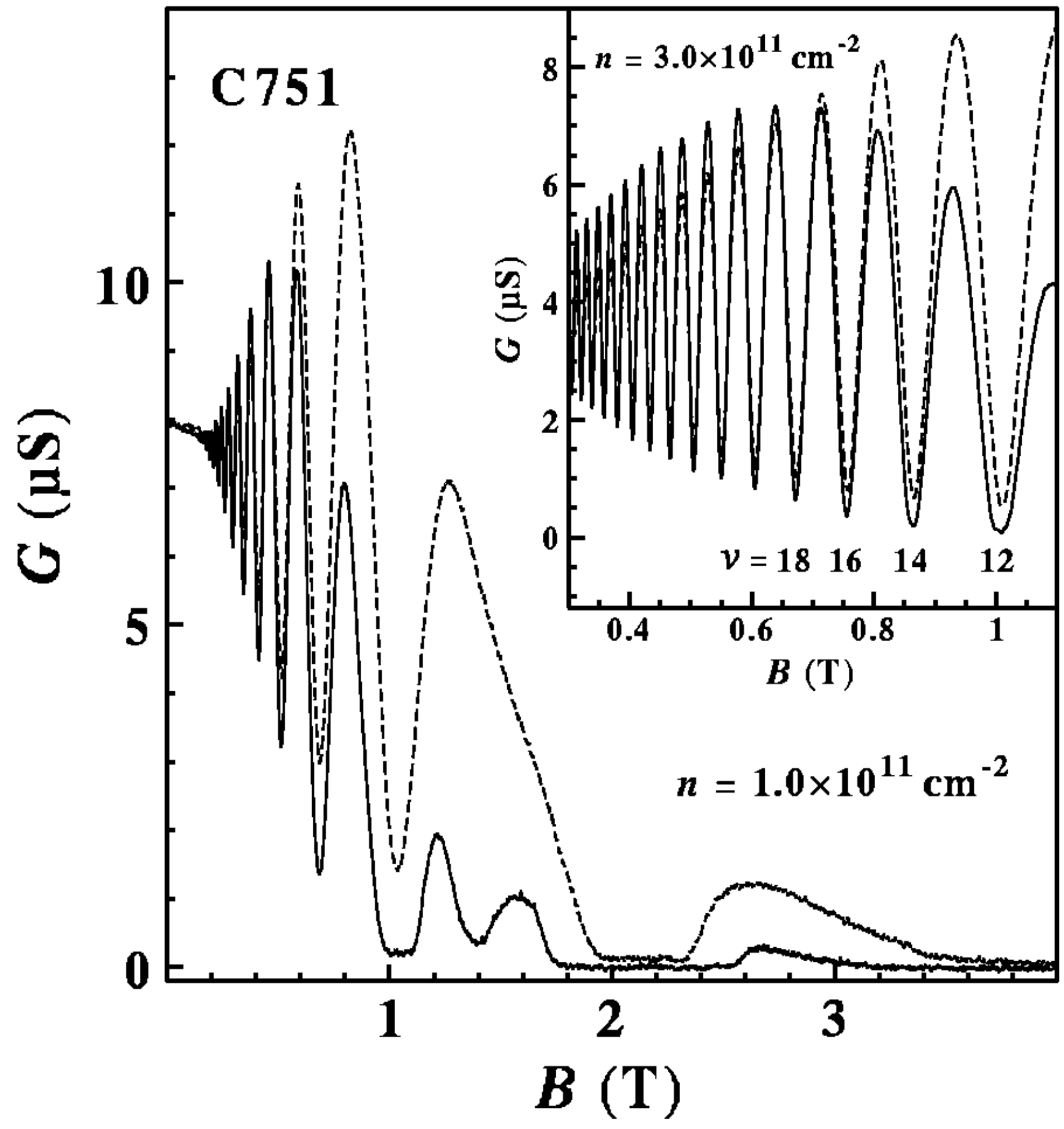


FIG 8

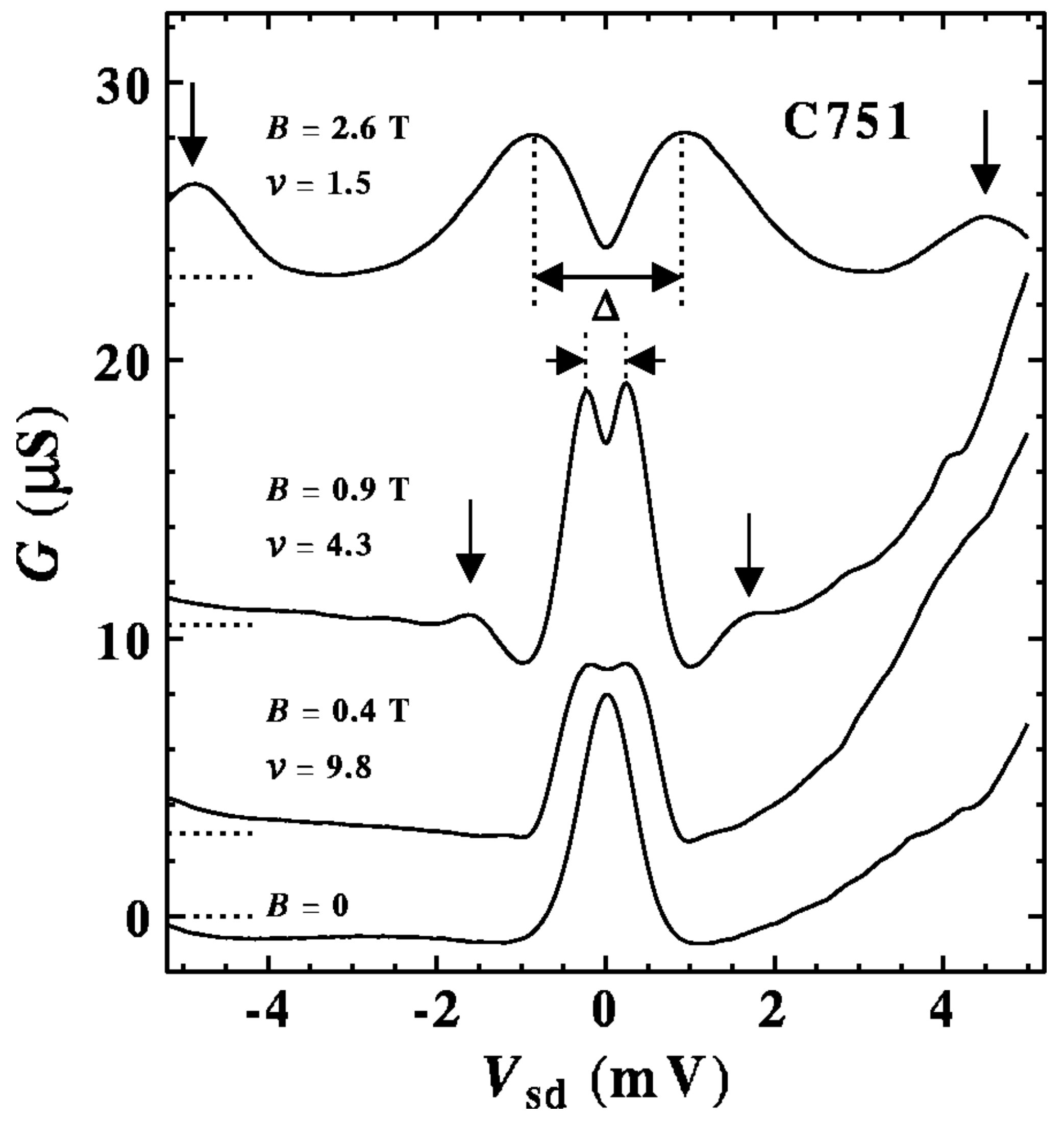




\section{FIG 9}

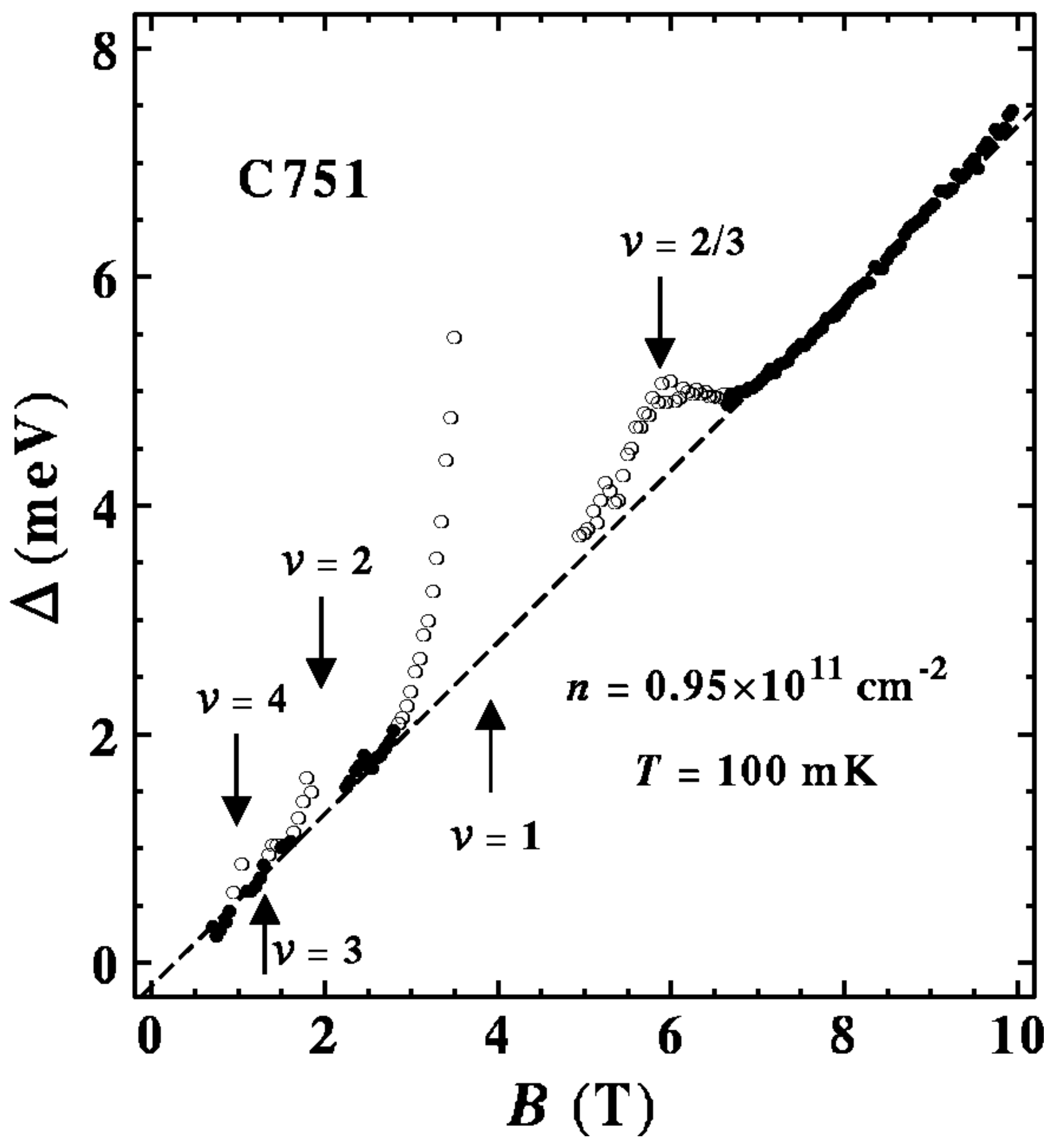




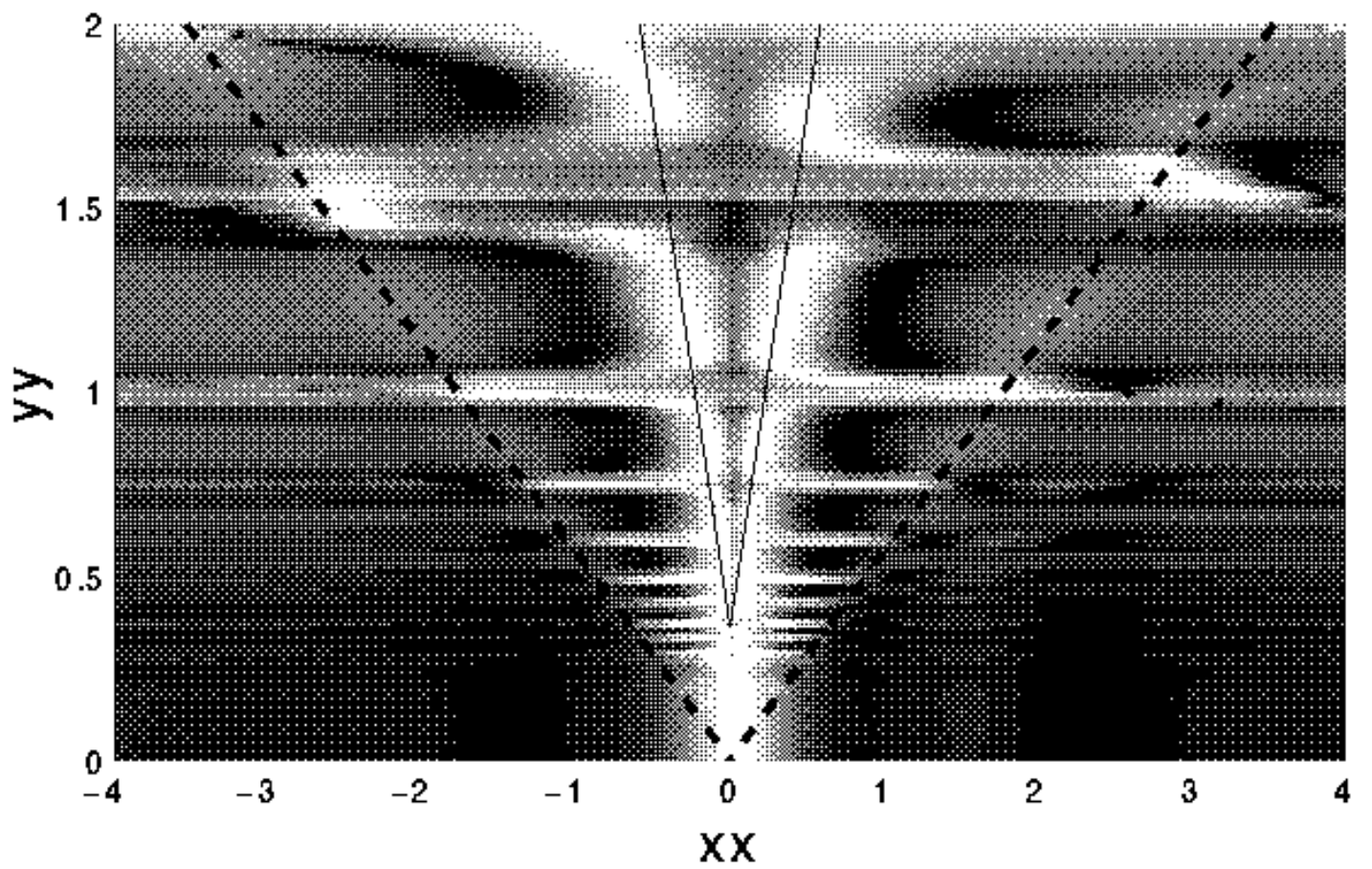




\section{FIG 11}

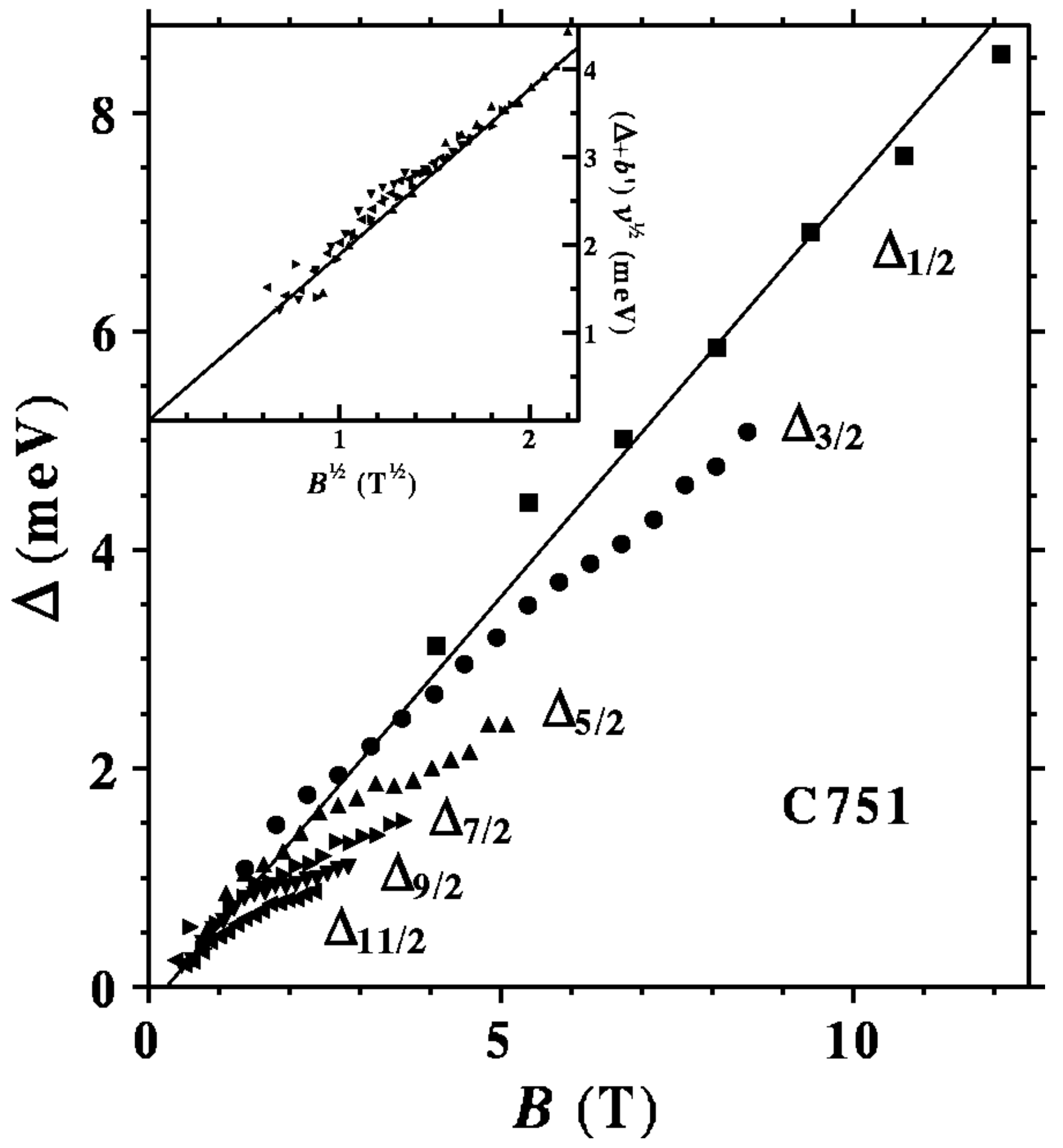



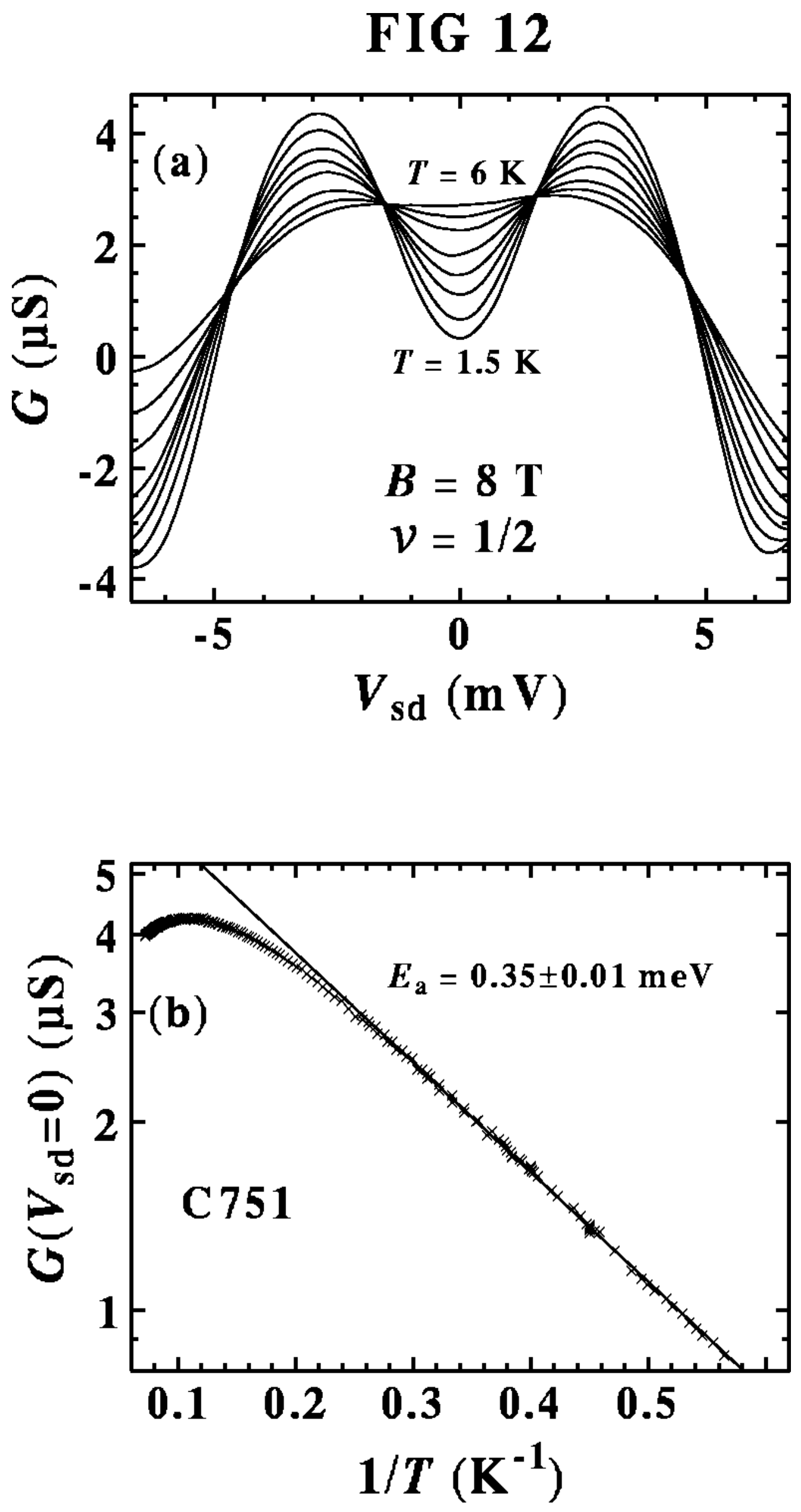


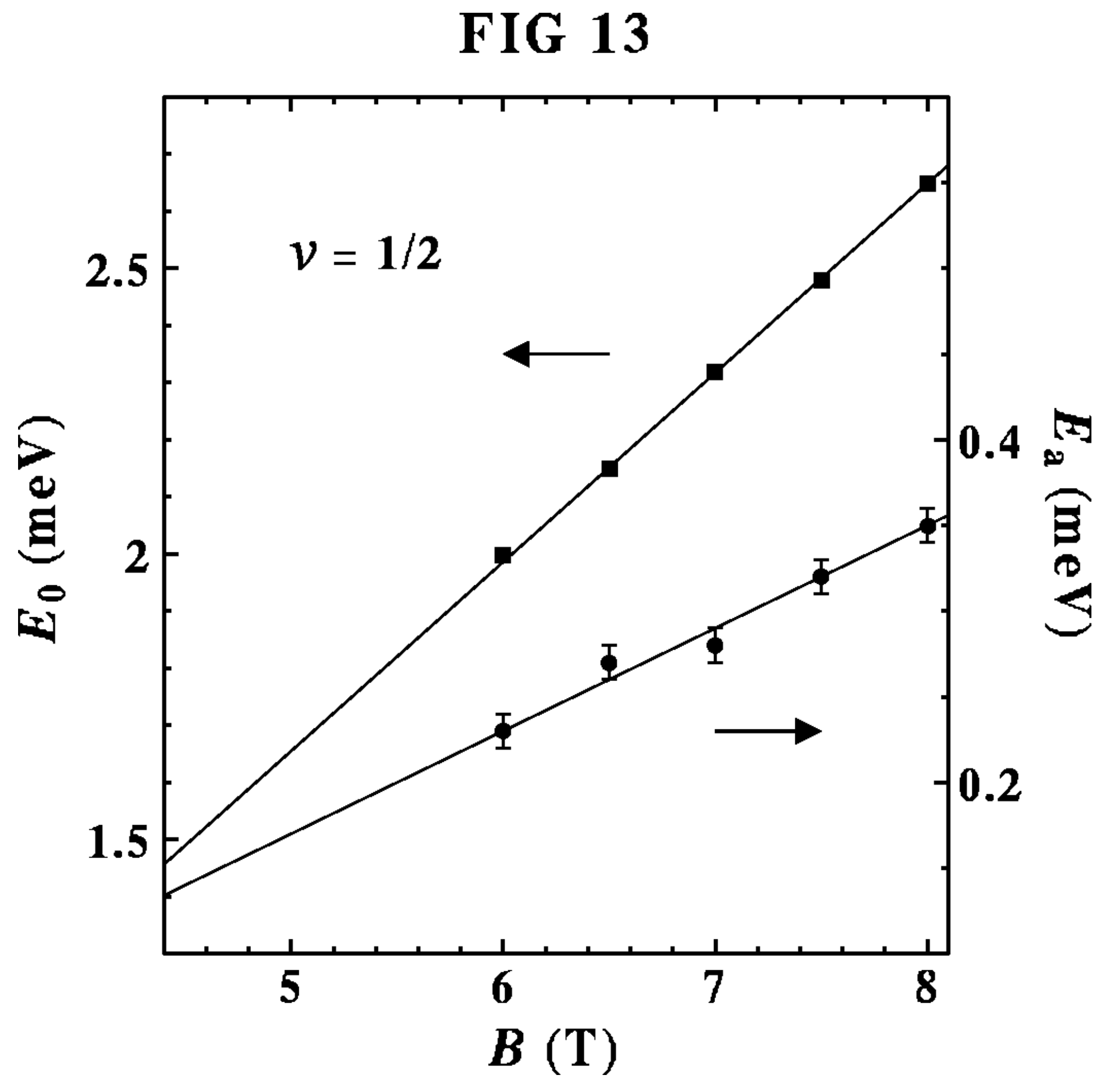




\section{FIG 14}
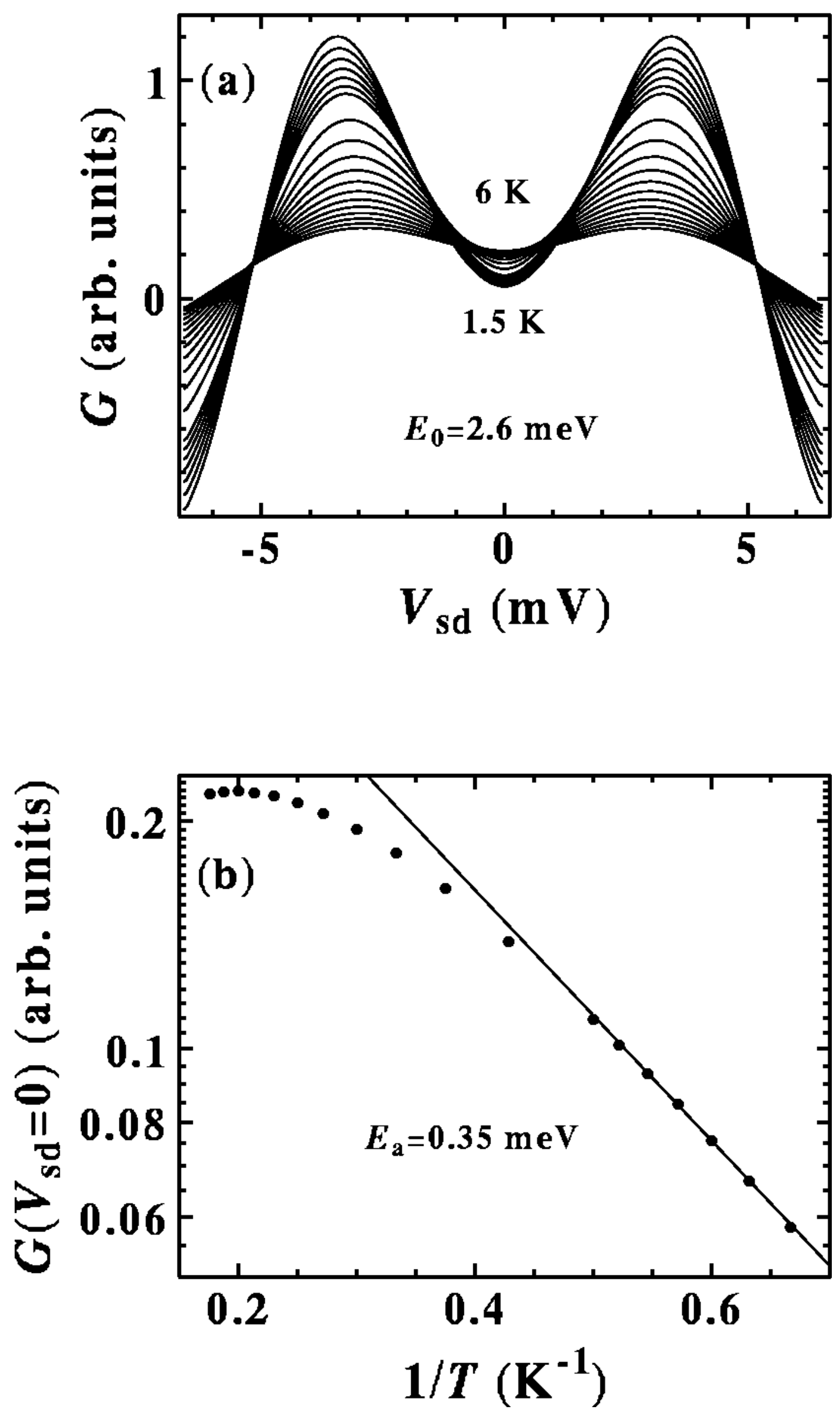